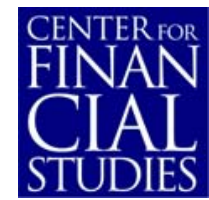

No. $2008 / 28$

Price Adjustment to News with Uncertain Precision

Nikolas Hautsch, Dieter Hess, and Christoph Müller 


\section{Center for Financial Studies}

The Center for Financial Studies is a nonprofit research organization, supported by an association of more than 120 banks, insurance companies, industrial corporations and public institutions. Established in 1968 and closely affiliated with the University of Frankfurt, it provides a strong link between the financial community and academia.

The CFS Working Paper Series presents the result of scientific research on selected topics in the field of money, banking and finance. The authors were either participants in the Center's Research Fellow Program or members of one of the Center's Research Projects.

If you would like to know more about the Center for Financial Studies, please let us know of your interest.

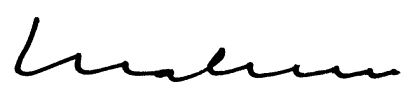

Prof. Dr. Jan Pieter Krahnen

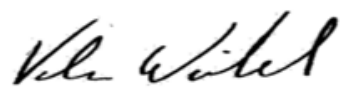

Prof. Volker Wieland, Ph.D. 


\title{
Price Adjustment to News with Uncertain Precision*
}

\author{
Nikolas Hautsch ${ }^{1}$, Dieter Hess ${ }^{2}$, \\ and Christoph Müller ${ }^{3}$
}

June 2008

\begin{abstract}
:
Bayesian learning provides the core concept of processing noisy information. In standard Bayesian frameworks, assessing the price impact of information requires perfect knowledge of news' precision. In practice, however, precision is rarely dis- closed. Therefore, we extend standard Bayesian learning, suggesting traders infer news' precision from magnitudes of surprises and from external sources. We show that interactions of the different precision signals may result in highly nonlinear price responses. Empirical tests based on intra-day Tbond futures price reactions to employment releases confirm the model's predictions and show that the effects are statistically and economically significant.
\end{abstract}

JEL Classification: E44, G14

Keywords: Bayesian Learning, Macroeconomic Announcements, Information Quality, Precision Signals

\footnotetext{
* For valuable comments we are grateful to participants of the DGF Annual Meeting 2007 in Dresden, the AFFI International Meeting 2007 in Paris, the MFA Annual Meeting 2008 in San Antonio, the Eastern Finance Association Meetings 2008 in St. Pete, FL, and seminar participants at the University of Konstanz. We are indebted to Kirk Mueller (BLS) and Raman Subri (DOL, ETA) for providing us with historical figures and background sampling statistics on the U.S. Employment Report and Unemployment Insurance Claims. Data on analysts' forecasts are obtained from Money Market Services (MMS) and Informa Global Markets. The first author gratefully acknowledges financial support from the EU Commission through MRTN-CT-2006-034270 COMISEF as well as from the Deutsche Forschungsgemeinschaft through the SFB 649 "Economic Risk". The third author gratefully acknowledges financial support from the Deutsche Forschungsgemeinschaft.

1 School of Business and Economics as well as CASE - Center for Applied Statistics and Economics, Humboldt-Universit“at zu Berlin, Quantitative Products Laboratory (QPL), Berlin, and Center for Financial Studies (CFS), Frankfurt. Address: Spandauer Str. 1, D-10178 Berlin, Germany, tel: +49 (0)302093 5711, email: nikolaus.hautsch@wiwi.hu-berlin.de

2 University of Cologne, Corporate Finance Seminar and Centre for Financial Research (CFR), Address: Albertus-Magnus-Platz, D-50923 Cologne, Germany, tel: +49 (0)221 470 7877, email: hess@wiso.uni-koeln.de 
Bayesian learning is a powerful concept, explaining why rational market participants may react differently to seemingly identical news: precise news moves prices more strongly than imprecise, noisy information. However, while it is typically assumed that the news' precision is known, in practice, it is rarely disclosed. Consequently, traders have to make a judgement on their own. Our analysis focuses on this lack of (perfect) precision knowledge: First, we extend standard Bayesian learning models to cases where traders have to gather precision estimates. A major implication of this extension is that price response coefficients - measuring the reaction of prices to news - strongly depend on traders' perception of information precision. This explains non-linear price reactions to unanticipated information. Second, we test this implication of non-constant price impact coefficients by analyzing high-frequency price responses in the U.S. T-Bond futures market to the U.S. employment report. ${ }^{1}$ Obtaining strong empirical evidence that instantaneous price reactions exhibit pronounced non-linearities which depend crucially on traders' perception of news' precision, we provide new insights into price discovery and market participants' learning behavior in an uncertain environment.

Standard Bayesian learning provides a clear answer how market prices react to information of differing quality - if this quality is perfectly known: (i) price reactions are linear in the amount of unanticipated information and (ii) the precision of new information relative to the precision of pre-announcement expectations determines how much market participants adjust their expectations. Hence, noisy (or diffuse) information has little impact on prices, whereas precise news moves market participants' expectations and hence prices more strongly. However, so far it is theoretically and empirically quite unclear how prices adjust in the much more realistic case when news' precision is unknown.

\footnotetext{
${ }^{1}$ Several studies show that among macroeconomic announcements, employment figures have by far the most pronounced impact on financial markets, in particular, on bond and foreign exchange markets (see, e.g., Andersen and Bollerslev, 1998, Fleming and Remolona, 1999, or Andersen, Bollerslev, Diebold and Vega, 2003).
} 
A first contribution to relax the usual assumptions of normally distributed variables with perfectly known parameters is provided by Subramanyam (1996). In his framework, traders try to infer the precision of news from a single noisy precision signal, i.e., the magnitude of the surprise component in an announcement. Due to this strict link between the expected precision and the signal magnitude, surprisingly large news may be interpreted as too good to be true. ${ }^{2}$ Consequently, price reactions are relatively strong for small surprises but relatively weak for big ones. This results in S-shaped price reactions to unexpected news. Nevertheless, a restrictive assumption in this setting is that market participants assess the reliability of news based solely on the released figures' magnitudes and ignore any other potentially available information on the news' precision. This is rather unrealistic in practice and can cause severe misinterpretations of the announced figures. Indeed, big surprises may very well be quite precise, and then, should generate strong price reactions.

Recent empirical evidence suggests that market participants consider different sources of information when assessing news' precision. For the employment report, Hautsch and Hess (2007) show that revisions of past headline figures provide information which can be exploited for such a purpose. Furthermore, they show that prices in the Tbond futures market indeed react more strongly to employment information when this revision-based precision estimate indicates a high information quality. Similarly, Pilotte and Manuel (1996) analyze price reactions in response to stock split announcements arguing that investor's confidence in the quality of a signal relies on their past experience. Furthermore, considering company reports, Sloan (1996), Feltham and Pae (2000), and Richardson, Sloan, Soliman and Tuna (2005), among others, show that accruals provide information about the quality of stated earnings. Overall, these studies suggest that

\footnotetext{
${ }^{2}$ See Mattsson, Voorneveld and Weibull (2007). In an early contribution, Milgrom (1981) studied this effect and provided conditions for monotonicity of price reactions in the announced information. These monotonicity results, based on a monotone likelihood ratio criterion, were recently generalized by Mattsson et al. (2007) in a discrete choice model under uncertainty.
} 
market participants may obtain precision signals by inspecting additional information related to a particular announcement.

Therefore, building on Subramanyam (1996) we develop a learning model allowing for uncertainty in news' precision where traders infer about the quality of information using two different sources as precision signals. First, so-called 'external' precision signals such as the sample size of a survey or the reputation of an auditing company - directly influence the perception of information precision. Second, the released information itself serves as an 'internal' precision signal. In accordance with Subramanyam (1996), this implies that the probability of news being imprecise increases with its magnitude (i.e., such news is believed to be too big to be true). We show that such learning behavior implies non-linear, S-shaped price response functions, i.e., the price response coefficient becomes smaller for big absolute surprises. Additionally, the model predicts stronger reactions to news which is perceived to be more precise given additional information. In this case, the S-shaped curvature of the price response function becomes even more pronounced and deviates strongly from linearity. We also show that our results both hold in a framework where the precision of the prior distribution is itself uncertain and are valid for a wide range of distributional assumptions.

In an empirical analysis of the price reactions of CBOT T-bond futures to the release of U.S. employment data, we provide strong evidence in favor of Bayesian learning under these two types of precision signals. From data revisions in employment releases, we extract release-specific external precision measures, which do not depend on the surprise itself. The estimated price response curves clearly reveal that prices (i) adjust non-linearly in response to unanticipated information with an S-shaped pattern and (ii) react significantly differently depending on the external precision signal. Also from an economic perspective, our results are strongly significant. We show that ignoring the available precision signals leads to severe estimation errors when determining the 
price impact of a news release.

The remainder of this paper is organized as follows. The following section presents a theoretical Bayesian learning framework which allows for the precision of arriving news to be unknown as well as for uncertainty in the prior distribution. Section 2 describes the high-frequency return data as well as the employment data and outlines the estimation procedure. The empirical results are presented and discussed in Section 3. Finally, Section 4 concludes.

\section{A Bayesian Learning Model}

\subsection{Standard Bayesian Learning}

Bayesian learning models provide a framework for analyzing how new information is incorporated into expectations and prices when prior information and incoming news contain uncertainty. Throughout our analysis we assume that all market participants are homogeneous and have the same information just before the release of some public announcement. The price $P$ of a risky asset is assumed to be proportional to traders' expectations of an economic variable $X$ with proportionality factor $\nu$, i.e. $P=\nu \cdot E[X]$. The beliefs on $X$ prior to the announcement are assumed to be normally distributed with known parameters, i.e. $X \sim N\left(\mu_{F}, 1 / \rho_{F}\right)$, where $\mu_{F}$ is the mean of the prior information on $X$ and $\rho_{F}$ denotes their precision, defined as the inverse of the variance. This prior information represents the market's conditional probability distribution of the variable $X$ given all available information, including, for example, all publicly released analysts' forecasts. Empirical research on the impact of scheduled announcements typically assumes that the distribution of prior beliefs in the market may be approximated by the distribution of analysts' forecasts. Hence, it is implicitly assumed that analysts' forecasts are unbiased for $X$, and, together with their cross-sectional dispersion, they 
provide a consistent estimate of the market's prior information. ${ }^{3}$

Now, say an announcement is released, providing a noisy estimate of $X$. It is assumed that the released figure includes an additive error, i.e., $A=X+\varepsilon$, where $\varepsilon$ is a zeromean normally-distributed error term with variance $\operatorname{Var}[\varepsilon]=1 / \rho_{\varepsilon}$ and $E[X \cdot \varepsilon]=$ 0 . Consequently, traders receive an unbiased estimate of the underlying variable $X$, whose precision is reflected by $\rho_{\varepsilon}$. The additive error term structure implies that the unconditional variance of the news release exceeds the variance of the market's prior information. Accordingly, the announcement $A$ is distributed as $A \sim N\left(\mu_{F}, 1 / \rho_{A}\right)$. After observing the public announcement, traders adjust their beliefs according to Bayes' rule. Traders' beliefs after the announcement are normally distributed with

$$
\mu_{P}:=E[X \mid A]=\mu_{F}+\left(A-\mu_{F}\right) \frac{\rho_{A}}{\rho_{F}}=\mu_{F}+\left(A-\mu_{F}\right) \frac{\rho_{\varepsilon}}{\rho_{F}+\rho_{\varepsilon}}
$$

and

$$
\rho_{P}:=\operatorname{Var}[X \mid A]^{-1}=\rho_{F}+\rho_{\varepsilon}
$$

Consequently, after traders observe the signal $A$, the market price of the risky asset changes as

$$
\Delta P=\nu \cdot\left(\mu_{P}-\mu_{F}\right)=\nu \cdot S \cdot \pi
$$

where $\pi$ denotes the so-called 'price-response coefficient'

$$
\pi:=\frac{\rho_{A}}{\rho_{F}}=\frac{\rho_{\varepsilon}}{\rho_{\varepsilon}+\rho_{F}} .
$$

Hence, the main model implication is that price changes are proportional to the surprise $S:=A-\mu_{F}$, where the proportionality factor $\pi$ depends on the relative precision of announcements and the market's forecast.

\footnotetext{
${ }^{3}$ See, e.g., Abarbanell, Lanen and Verrecchia (1995), Mohammed and Yadav (2002), Andersen, Bollerslev, Diebold and Vega (2003) and Hautsch and Hess (2007).
} 


\subsection{Surprises as an 'Internal' Signal of the Precision of Releases}

Announcements such as employment figures are usually released without an associated precision measure, which contradicts the assumptions of the standard Bayesian learning model. Therefore, Subramanyam (1996) relaxes this framework by treating news' precision to be unknown and assuming that the announcement is conditionally normally distributed given the true precision, i.e., $A \mid \rho_{A} \sim N\left(\mu_{F}, \rho_{A}\right)$. Formally, Bayesian updating of traders works in a way similar to the basic framework, yielding

$$
\mu_{P}=E[X \mid A]=\mu_{F}+\left(A-\mu_{F}\right) \frac{E\left[\rho_{A} \mid A\right]}{\rho_{F}}=\mu_{F}+S \cdot \pi(S)
$$

with $E\left[\rho_{A} \mid A\right]$ representing traders' conditional expectation of the signal's precision given its realization. Hence, it turns out that the price response coefficient $\pi(S)$ is no longer constant but rather depends on the absolute surprise. Consequently, the latter serves as an 'internal' signal of news' precision. As shown by Subramanyam (1996) and illustrated in a more general framework in the next section, this generates a nonlinear relationship between the magnitude of the surprise and the implied update of traders' beliefs. In particular, if traders observe high absolute values of unanticipated information, they conclude that these stem from an announcement with low precision. This reduces their adjustment of beliefs in absolute terms, which in the extreme case may even generate negative marginal contributions of surprises, resulting in an S-shaped price response curve. However, this direct link between the amount of unanticipated information and the expected precision is relatively restrictive, since it implies that big surprises are always too big to be true. But in an environment where information precision is high, occasionally, big surprises may occur as well. In such cases, we would expect to observe stronger price reaction, which is ruled out in this setup. 


\section{3 'External' Signals of the Precision of Releases}

Extending the previous setting, we assume that traders not only evaluate the released information itself when assessing news' precision but also employ other data sources. For instance, for the U.S. employment report, Hautsch and Hess (2007) show that traders may infer the precision of announced employment figures by inspecting the time series of historical revisions of the headline figure. Since revisions in announcements reflect (ex post-) sampling errors, a natural precision measure arises from their conditional variance.

Suppose that market participants are able to observe a so-called external signal $\hat{\rho}_{A}$ for the precision of the announcement $\rho_{A}$. Here, 'external' refers to cases where the signal is not directly linked to the announced figure itself. For example, $\hat{\rho}_{A}$ might be information on the sample size of a survey, the reliability of data collection or a precision estimate based on (past) revisions, as in Hautsch and Hess (2007).

Let this additional precision signal follow a conditionally normal distribution given the true precision $\rho_{A}$, i.e., $\hat{\rho}_{A} \mid \rho_{A} \sim N\left(\rho_{A}, \sigma_{\hat{\rho}_{A}}^{2}\right)$. Moreover, we assume that the announcement $A$ and the precision signal $\hat{\rho}_{A}$ are conditionally independent given the true precision $\rho_{A}$. Therefore, the precision signal and the news release are only linked indirectly via the true precision. If $\sigma_{\hat{\rho}_{A}}^{2}$ reaches zero, the signal reveals the true precision of the announcement. In this case, the surprise itself no longer serves as an internal precision signal, and we are back to the standard Bayesian learning model. If $\sigma_{\hat{\rho}_{A}}^{2}$ is different from zero, both precision signals are taken into account by market participants. In a way analogous to the updating equations given above, traders form their beliefs as ${ }^{4}$

$$
\mu_{P}=E\left[X \mid A, \hat{\rho}_{A}\right]=\mu_{F}+\left(A-\mu_{F}\right) \frac{E\left[\rho_{A} \mid A, \hat{\rho}_{A}\right]}{\rho_{F}}=\mu_{F}+S \cdot \pi\left(S, \hat{\rho}_{A}\right) .
$$

\footnotetext{
${ }^{4}$ For a formal derivation, see Appendix A.
} 
As before, adjustments in traders' beliefs depend symmetrically on the amount of surprise associated with the news. However, now the market incorporates additional information into its price formation. This is reflected by the price response coefficient $\pi(\cdot)$ depending not only on $S$ but also on $\hat{\rho}_{A}$. As shown in Appendix A, the conditional expectation of precision $E\left[\rho_{A} \mid A, \hat{\rho}_{A}\right]$ is computed by

$$
E\left[\rho_{A} \mid A, \hat{\rho}_{A}\right]=\frac{\int_{S_{A}} \rho_{A} f\left(A \mid \rho_{A}\right) f\left(\hat{\rho}_{A} \mid \rho_{A}\right) f\left(\rho_{A}\right) d \rho_{A}}{\int_{S_{A}} f\left(A \mid \rho_{A}\right) f\left(\hat{\rho}_{A} \mid \rho_{A}\right) f\left(\rho_{A}\right) d \rho_{A}},
$$

where $f(\cdot)$ denote the corresponding conditional and unconditional p.d.f.'s and the support $S_{A}$ of $f\left(\rho_{A}\right)$ is given by $S_{A} \in\left(\rho_{F}, \infty\right)$. Hence, it turns out that the expected precision depends not only on $\hat{\rho}_{A}$ and $A$ but also on the unconditional prior distribution of the precision, $f\left(\rho_{A}\right)$.

In Proposition 1, we will show in accordance with Subramanyam (1996) that the amount of unanticipated information influences the expected precision of the announcement negatively. This result holds irrespective of the choice of the underlying prior distribution $f\left(\rho_{A}\right)$ :

Proposition 1 The price response coefficient $\pi\left(S, \hat{\rho}_{A}\right)$ is strictly decreasing in the absolute magnitude of the surprise $|S|$ for any prior distribution $f\left(\rho_{A}\right)$, i.e., $\partial \pi\left(S, \hat{\rho}_{A}\right) / \partial|S|<0$.

\section{Proof: See Appendix A.}

Therefore, prices react relatively strongly to less surprising news and relatively weakly to news with a high element of surprise. Consequently, there are two effects determining the change in beliefs $\left(\mu_{P}-\mu_{F}\right)$ after an announcement is made: First, given the price response coefficient $\pi(\cdot)$, a big (small) surprise $S=A-\mu_{F}$ strengthens (weakens) the price reaction linearly. Second, according to Proposition 1, a big (small) surprise 
decreases (increases) the expected signal precision and thus decreases (increases) $\pi(\cdot)$. As shown in Proposition 2, the latter effect induces price reactions which are S-shaped in absolute surprises:

Proposition 2 The marginal impact of the surprise $S$ on investors' updates of beliefs, $\mu_{P}-\mu_{F}$, is given by $\partial\left(\mu_{P}-\mu_{F}\right) / \partial S=\pi\left(S, \hat{\rho}_{A}\right)-S^{2} \rho_{F}^{-1} \operatorname{Var}\left[\rho_{A} \mid A, \hat{\rho}_{A}\right]$.

Proof: See Appendix A.

Hence, investors marginally update their expectations in the direction of the surprise as long as $\pi\left(S, \hat{\rho}_{A}\right)-S^{2} \rho_{F}^{-1} \operatorname{Var}\left[\rho_{A} \mid A, \hat{\rho}_{A}\right]>0$. However, if $|S|$ becomes large, the relationship may reverse and the marginal effect of absolute surprises may become negative, i.e., $\partial\left(\mu_{P}-\mu_{F}\right) / \partial S<0$. These effects are enforced if $\rho_{F}$ is small and $\operatorname{Var}\left[\rho_{A} \mid A, \hat{\rho}_{A}\right]$ is large. Consequently, we obtain an S-shaped price reaction, as graphically illustrated in Figure 1. Note that in the case of a degenerated prior distribution $f\left(\rho_{A}\right)$, we get a linear response, as in the basic model. Hence, the result of an S-shaped relationship between surprises and traders' updates of expectations according to Subramanyam (1996) still holds in this extended framework.

However, the following proposition shows that traders' conditional expectations of news' precision depend positively on the external precision signal $\hat{\rho}_{A}$. Hence, traders update their conditional expectations more strongly (less strongly) if $\hat{\rho}_{A}$ increases (decreases). Consequently, $\hat{\rho}_{A}$ affects the price response coefficient $\pi\left(S, \hat{\rho}_{A}\right)$ in the opposite direction of $|S|$.

Proposition 3 The price response coefficient $\pi\left(S, \hat{\rho}_{A}\right)$ and the absolute signal response $\left|\mu_{F}-\mu_{P}\right|$ are strictly increasing in the observed value of the precision signal $\hat{\rho}_{A}$ for any prior distribution $f\left(\rho_{A}\right)$, i.e., $\partial \pi\left(S, \hat{\rho}_{A}\right) / \partial \hat{\rho}_{A}>0$ and 
$\partial\left|\mu_{p}-\mu_{F}\right| / \partial \hat{\rho}_{A}>0$

Proof: See Appendix A.

The proposition also states that a central implication of standard Bayesian learning is maintained even if the true precision parameter of the news is replaced by a noisy signal: market prices react more strongly to news which is perceived to be more precise, whereas news which appears to be imprecise induces rather moderate market reactions. However, as shown in Figure 1, the existence of an 'external' precision measure $\hat{\rho}_{A}$ induces an additional effect which amplifies the S-shape. A higher $\rho_{A}$ leads to a flattening of the price response curve for small surprises near zero but to more pronounced S-shaped price responses to big surprises.

\subsection{Accounting for Uncertainty in the Prior Distribution}

So far, we have assumed that traders have normally-distributed prior beliefs on the distribution of the variable $X$ with perfectly known parameters. However, traders generate their views by, for example, relying on analysts' forecasts; thus, they face estimation errors. In practice, traders might approximate the precision of prior information by the dispersion of different analysts' forecasts. However, the quality of such estimates is itself subject to uncertainty. In order to capture uncertainty in the precision of prior information, we assume that this precision is random and follows a distribution $f\left(\rho_{F}\right)$. Then, $X$ is assumed to be conditionally normally distributed given $\rho_{F}$, i.e. $X \mid \rho_{F} \sim N\left(\mu_{F}, 1 / \rho_{F}\right)$. This results in a scale mixture distribution for the prior distribution, yielding

$$
f(X)=\int_{S_{F}} f\left(X \mid \rho_{F}\right) f\left(\rho_{F}\right) d \rho_{F}
$$

with $S_{F} \in(0, \infty)$. Nevertheless, as shown in Proposition 4, all previous results still hold: 
Proposition 4 If the prior distribution of traders follows a scale mixture of normal distributions, Propositions 1, 2 and 3 still hold.

Proof: See Appendix A.

Obviously, this proposition states that our analysis is not restricted to cases with normally-distributed variables but holds for a wide class of distributions, including fat-tailed prior distributions.

\subsection{Testable Implications of the Model}

The learning model outlined above yields hypotheses on how traders' expectations adjust to new information. Assuming that prices are proportional to traders' expectations of the observed market variable, the following testable hypotheses arise:

(1) The standard Bayesian learning model with perfectly-known normal distributions presented in Section 11.1 implies a linear price response function,

$$
\Delta P=\nu \cdot S \cdot \pi
$$

Here, a higher magnitude of surprise implies higher absolute price reactions, since the price response coefficient $\pi$ is a constant and known parameter which does not depend on the revealed unanticipated information, $S$. The price response coefficient, then, is determined by the precision of the announcement and the precision of the released data.

(2) As shown in Section 11.2, the model suggested by Subramanyam (1996) implies

$$
\Delta P=\nu \cdot S \cdot \pi(S) .
$$


Here, news precision is unknown and is thus inferred from the magnitude of surprises. Since large surprises serve as a signal for a low precision of news, the price response coefficient $\pi(S)$ decreases in the absolute size of the surprise $|S|$, implying an S-shaped relationship between $\Delta P$ and $S$.

(3) Allowing for additional external precision signals $\hat{\rho}_{A}$ as in Section 11.3, we get

$$
\Delta P=\nu \cdot S \cdot \pi\left(S, \hat{\rho}_{A}\right)
$$

The previous result of an S-shaped price response curve still holds, but we observe the additional effect of a positive relationship between $\Delta P$ and $\hat{\rho}_{A}$. In this case, both effects might work in opposite directions and the S-shape of the price response curve is amplified if news' precision is high.

\section{Data and Empirical Framework}

\section{$2.1 \quad$ Data}

The model implications outlined above will be empirically tested in the following sections. Note that we do not estimate this model in a structural way, since this would require additional structural assumptions in order to estimate $E\left[\rho_{A} \mid A, \hat{\rho}_{A}\right]$. We rather test for the implications summarized above in reduced form by estimating the shape of the price reaction curve in response to $S$ and the perceived precision of news $\left|\hat{\rho}_{A}\right|$. We use intraday returns of CBOT T-bond futures corresponding to one of the most liquid futures markets as well as monthly releases of the U.S. employment report. The latter is by far the most influential scheduled macroeconomic release, and its impact on financial markets is investigated in a wide range of studies. ${ }^{5}$ While the employment

\footnotetext{
${ }^{5}$ Several empirical studies provide evidence that unanticipated information in the employment report has a strong influence not only on bond market prices (e.g. Becker, Finnerty and Kopecky (1996), Fleming and Remolona (1999c), and Hautsch and Hess (2002)) but also on foreign exchange rates
} 
report contains detailed information on the employment situation in the U.S., market participants focus in particular on two headline figures: the nonfarm payrolls figure and the unemployment rate figure. The disclosure of this information offers a rare opportunity to analyze Bayesian learning effects in the adjustment of price in response to news, since both the amount of unanticipated information and a release-specific precision measure can be obtained.

Hautsch and Hess (2007) document the importance of news' precision in a framework where traders are assumed to use external information to make inferences about the precision of news. To facilitate a comparison with these results, we employ a similar data set based on two-minute log returns of T-bond futures in 90-minute windows around employment announcements. ${ }^{6}$ However, our dataset covers an extended sample period of 15 years, from January 4th, 1991 to December 2nd, 2005. These high frequency T-bond data are obtained from the Chicago Board of Trade (via their Time \& Sales records). From our sample period we obtain 161 event windows in which no other major information event occurs aside from the release of the employment report. ${ }^{7}$ Thus information processing during these event windows is driven only by employment figures. Like previous studies, we use so-called consensus estimates, i.e., medians of analysts' forecasts, to approximate the anticipated part of information in the employment headline figures. These analysts' forecasts are obtained from Informa Global Markets (e.g. Hardouvelis (1988), Andersen et al. (2003)) and stock prices (e.g. Boyd, Hu and Jagannathan (2005)).

${ }^{6} \mathrm{Log}$ returns are calculated on the basis of the last trading price observed during a two-minute interval. We use the same time window, 8:22-9:52 a.m. EST. Since trading starts at 8:20, the first return can be calculated for the interval 8:22-8:24. In order to avoid the influence of other announcements, released at 10:00 a.m. EST., only price observations up to 9:52 a.m. EST are used. Like most previous studies, we focus on the front month contract, i.e., the most actively traded contract among the nearby and second nearby contracts.

${ }^{7}$ We eliminate 15 days in which other reports were released during our 90-minute window, particularly releases of Leading Indicators, Personal Income, and Gross Domestic Product. Furthermore, we eliminate one inadvertently early employment release in November 1998 (Fleming and Remolona 1999b) and another three releases which were presumably affected by the temporary shutdown of federal agencies due to the budget dispute during the Clinton administration (see Hess, 2004). This leaves us with a total of 161 observations. 
(formerly S\&P Money Market Services, MMS). The announcement data are extracted from the original, unrevised employment releases from the Bureau of Labour Statistics (BLS). In accordance with other studies, we concentrate on the headline information in the employment report, i.e., surprises in the nonfarm payrolls figure $S_{N F}$ and the unemployment rate $S_{U N} \cdot{ }^{8}$ Note that nonfarm payrolls are revised in the subsequent month. We include this revision information, $R_{N F, m}$, into our analysis. In order to facilitate a direct comparison across the information components, all surprise and revision variables are measured in percentage changes.

In order to extract release-specific precision measures for the monthly employment releases, we employ the procedure suggested by Hautsch and Hess (2007). First, as a precision measure for prior information, the dispersion of analysts' forecasts before an announcement is used. ${ }^{9}$ In particular, the standard deviation of analysts' forecasts $\hat{s}_{F, m}$ for a particular month $m$ is interpreted as a measure of the cross-sectional dispersion of expectations and serves as a proxy for the precision of prior information, i.e. $\hat{\rho}_{F, m}=1 / \hat{s}_{F, m}^{2}$. Second, in order to obtain a measure for the precision of the announced information itself, a one-step-ahead prediction of the (conditional) variance of revisions is used. Using revisions in nonfarm payrolls is based on the idea that a large revision of the previous month's figure (as provided in the current report) indicates that the precision of that figure has obviously been poor. Hautsch and Hess (2007) illustrate that the magnitude of revisions, and thus the size of estimation errors in announced figures, are autocorrelated. Hence, the size of revisions as a proxy for news' precision is predictable. Corresponding forecasts are obtained from an

\footnotetext{
${ }^{8}$ The unanticipated information contained in the releases of month $m$ is then measured as the difference between the announced figure $A_{., m}$ and its median forecast $\mu_{F, ., m}$. For instance, the surprise in a nonfarm payrolls figure, $S_{N F, m}$, is determined as $S_{N F, m}=A_{N F, m}-\mu_{F, N F, m}$.

${ }^{9}$ This is in accordance with Abarbanell et al. (1995), Mohammed and Yadav (2002), Andersen et al. (2003) and Hautsch and Hess (2007), among others. However, note that the information set of all publicly available prior information may be even much larger. Furthermore, as Ottaviani and Sorensen (2006) argue, forecasts may be announced strategically depending on the forecaster's loss function, e.g. as the median of a distribution.
} 
ARMA-GARCH model fitted to the time series of revisions. Then, $\hat{\rho}_{\varepsilon, m}$ is obtained by $\hat{\rho}_{\varepsilon, m}=\widehat{\operatorname{Var}}\left[R_{N F, m} \mid R_{N F, m-1}, R_{N F, m-2}, \ldots\right]^{-1} .^{10}$

In order to reduce the impact of estimation noise on the quantification of news' precision and avoid the need to impose additional assumptions on the functional relationship between the precision measure and the induced price reaction, we restrict our analysis to a distinction between precise and imprecise news. These two states are identified based on a proxy of the price response coefficient $\hat{\pi}_{m}=\hat{\rho}_{\varepsilon, m} /\left(\hat{\rho}_{\varepsilon, m}+\hat{\rho}_{F, m}\right)$. We define news to be precise if $\hat{\pi}_{m}$ is equal to or above its sample median and imprecise otherwise. Estimating the relationship between price changes, the surprise $S$ and the derived precision dummy allows us to test for the implications of the generalized Bayesian learning framework outlined above.

\subsection{Specification of Price Response Curves}

Using two-minute $\log$ returns $r_{t}$ in the described 90-minute-windows around the employment release we estimate alternative ARMA-ARCH specifications augmented with appropriate sets of explanatory variables $x_{t}$, i.e.,

$$
r_{t}=c+\sum_{j=1}^{p_{1}} \phi_{1, j} r_{t-j}+\sum_{j=1}^{q_{1}} \phi_{2, j} \varepsilon_{t-j}+x_{t}^{\prime} \beta+\varepsilon_{t}, \quad \varepsilon_{t} \sim N\left(0, h_{t}\right),
$$

with

$$
h_{t}=\omega+\sum_{j=1}^{p_{2}} \psi_{1, j} \varepsilon_{t-j}^{2}+z_{t}^{\prime} \theta .
$$

Here, $t$ indexes the two-minute intervals around the release of the employment report for a given month $m$. In particular, $t=0$ indicates the interval immediately following the announcement, i.e. 8:30 - 8:32 a.m. EST and $t=1$ denotes the 8:32 - 8:34 interval. For simplicity, the index $m$ is suppressed.

\footnotetext{
${ }^{10}$ For more details, see Hautsch and Hess (2007).
} 
The conditional variance equation (10) captures ARCH effects. In addition, $z_{t}$ (with corresponding parameter vector $\theta)$ consists of regressors $\{\bar{t}, \sin (2 \cdot r \cdot \pi \cdot \bar{t}), \cos (2 \cdot r$. $\pi \cdot \bar{t})\}$ with $r=1, \ldots, R$ associated with a Fourier series approximation of order $R$ defined over the interval $\bar{t} \in(0,1)$ capturing the used 90-minutes window around the announcement. This allow us to control for (deterministic) seasonal volatility patterns around news releases. Preliminary studies show that such a specification captures most of the variation in conditional variances during the analyzed 90-minute interval. ${ }^{11}$

To test for the different implications of the Bayesian learning model discussed above, we use alternative specifications of the vector $x_{t}$. In particular, to impose the standard Bayesian learning model in accordance with Section 11.1, a dummy variable $D_{8: 30}$ indicating the interval 8:30 - 8:32 and a linear term for the surprise $D_{8: 30} \cdot S_{N F}$ are included (among other variables) as regressors in $x_{t}$ :

$$
x_{t}^{\prime}=\left[\ldots, D_{8: 30}, D_{8: 30} \cdot S_{N F}, \ldots\right] \text {, }
$$

where $S_{N F, m}$ contains the unexpected component in the nonfarm payrolls figure for month $m$. Obviously this specification implies a linear price relationship between nonfarm payroll surprises and the implied return.

In order to capture the impact of an internal precision signal (in accordance with Section 11.2), we allow for non-linear price responses to news in nonfarm payrolls by including power functions of this figure into the set of explanatory variables. To keep the model tractable, we allow for this flexibility only in the interval from 8:30-8:32, where most of the price movements after employmant announcements typically are realized. Correspondingly, we model the impact of surprises in nonfarm payrolls based on the regressors

$$
x_{t}^{\prime}=\left[\ldots, D_{8: 30}, D_{8: 30} \cdot S_{N F}, D_{8: 30} \cdot S_{N F}^{2}, D_{8: 30} \cdot S_{N F}^{3}, \ldots\right] .
$$

\footnotetext{
${ }^{11}$ Nevertheless, there might be heteroscedasticity components which are still ignored in our specification. Therefore, we use robust standard errors in accordance with Bollerslev and Wooldridge (1992).
} 
To estimate the most general (unrestricted) model allowing for both internal and external precision signals (in accordance with Section 11.3) we differentiate between precise and imprecise announcements by interacting the corresponding regressors with a dummy variable $D^{\text {klow }}$ which takes on a value of one if the external precision signal is below its sample median and zero otherwise:

$$
\begin{aligned}
x_{t}^{\prime}=\quad & {\left[\ldots, D_{8: 30} \cdot D^{\pi l o w}, D_{8: 30} \cdot D^{\pi h i g h},\right.} \\
& D_{8: 30} \cdot S_{N F} \cdot D^{\pi l o w}, D_{8: 30} \cdot S_{N F} \cdot D^{\pi h i g h}, \\
& D_{8: 30} \cdot S_{N F}^{2} \cdot D^{\pi l o w}, D_{8: 30} \cdot S_{N F}^{2} \cdot D^{\pi h i g h}, \\
& \left.D_{8: 30} \cdot S_{N F}^{3} \cdot D^{\pi l o w}, D_{8: 30} \cdot S_{N F}^{3} \cdot D^{\pi h i g h}, \ldots\right],
\end{aligned}
$$

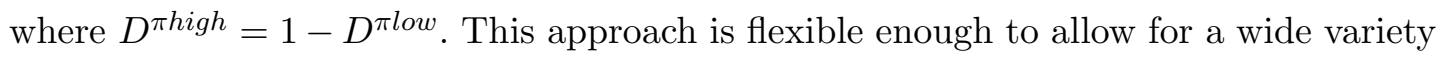
of shapes of the price response function. Starting with the linear specification, the conventional constant price impact coefficient is obtained as a reference case. Increasing the order of included polynomials allows us to test whether more non-linear terms are needed to describe the price response function appropriately. In addition, by interacting these terms with dummy variables indicating a low or high value of the external precision signal, we can analyze whether the shapes of the price response functions differ and thus gain insights regarding the relative weight, market participants place on internal and external precision signals.

In order to keep the model parsimonious and tractable, we mainly concentrate on the price response induced by announcements in nonfarm payrolls, which are by far the most influential macroeconomic headline figure. 


\section{Empirical Results}

Our empirical analysis proceeds in two steps. First, we will analyze whether we find significant evidence for S-shaped price response functions in accordance with Section 11.2. Second, we will investigate the impact of external precision signals on the strength and the shape of the price response in line with Section 11.3.

\subsection{Non-linearities in Price Response due to Internal Precision Sig- nals}

Table 1 reports estimation results based on five different specifications of equation (9). The lag order of the autoregressive components is chosen according to the Bayes information criterion (BIC) and reveals an $\mathrm{AR}(2)-\mathrm{ARCH}(3)$ specification as the preferred model. Aside from the variables discussed in the previous section, the conditional mean function includes additional variables consisting of surprises in the unemployment rate $S_{U N}$ as well as revisions in the nonfarm payrolls figure $R_{N F}$. Moreover, we allow for potential information leakage effects in the interval 8:28-8:30 as well as lagged price responses in the interval 8:32-8:34.

As a starting point, specification (A) provides estimation results for a basic model that does not account for any release-specific precision of unanticipated information. The results confirm several major findings of previous studies: ${ }^{12}$ First, the large values of the highly significant coefficients of $D_{8: 30} \cdot S_{N F}$ and $D_{8: 30} \cdot S_{U N}$ show that surprising headline information has a strong and significant impact on intraday returns. The directions of observed price reactions are consistent with standard theory, i.e., T-bond futures prices rise in response to 'good' news from the factor labor, i.e., a lower than

\footnotetext{
${ }^{12}$ See, for example, Becker et al. (1996), Balduzzi, Elton and Green (2001), Fleming and Remolona (1999a, b, c), or Hautsch and Hess (2002) for bond markets and Almeida, Goodhart and Payne (1998) or Andersen et al. (2003) for foreign exchange markets.
} 
expected increase in nonfarm payrolls and a higher than expected unemployment rate. Second, markets process unanticipated headline information very rapidly. As indicated by the insignificant coefficient of $D_{8: 32} \cdot S_{U N}$ and the relatively small coefficient of $D_{8: 32} \cdot S_{N F}$ (as compared to $D_{8: 30} \cdot S_{N F}$ ), the price reaction is completed within two to four minutes.

Specifications (B) - (E) allow for non-linearities in price responses. Specifically, the variables capturing the immediate price impact of unanticipated information in the nonfarm payrolls figure $D_{8: 30} \cdot S_{N F}$ are included as polynomial terms up to order three. Note that the theoretical Bayesian learning model with uncertain news' precision suggests that price reactions are symmetric around zero. Nevertheless, the imposed polynomials also allow for non-symmetric price responses. In particular, previous empirical studies suggest asymmetric effects of 'good' and 'bad' news in response to information releases. ${ }^{13}$

Specification (B) shows estimation results for a quadratic specification of the price response, while specification (C) includes terms up to the third order. Corresponding likelihood ratio (LR) tests clearly reject the linear specification (A) in favor of the non-linear models. Hence, higher order terms provide additional explanatory power for price responses to unanticipated information in the nonfarm payroll figure. On a $1 \%$ level, the more parsimonious specification (C) with terms up to the third order may not be rejected in favor of (D) and (E). Overall, in line with the LR tests, the Bayesian information criterion (BIC) suggests that specification (C) explains price responses best.

The results imply that the standard Bayesian learning model with a constant price response to unanticipated information can clearly be rejected. As an illustration, Figure

\footnotetext{
${ }^{13}$ See, e.g. Conrad, Cornell and Landsman (2002), Andersen et al. (2003) and Hautsch and Hess (2007)
} 
2 shows the estimated price-response curve to releases of the nonfarm payroll figure under specification (C). We find clear evidence for an S-shaped price response where price reactions to big surprises are relatively weaker than reactions to small surprises. This suggests that market participants evaluate the amount of unanticipated information contained in an announcement as an internal signal of information precision confirming the model by Subramanyam (1996).

\subsection{External Precision Signals and the Strength of the Price Response}

In order to investigate the impact of the external precision measure $\hat{\rho}_{A}$, we split the variable $D_{8: 30} \cdot S_{N F}$ (including higher order terms) into interactions with the dummy variables $D^{\pi h i g h}$ and $D^{\text {klow }}$ accounting for high vs. low values of $\pi_{m}$.

The estimation results based on alternative specifications of the immediate price response function are given in Table 2. The results for specification (F) confirm the findings in Hautsch and Hess (2007) that more precise information leads to significantly stronger price adjustments. Note that this base case does not account for nonlinear price adjustments but does imply a linear price reaction, as graphically illustrated in Figure 3. A comparison of the goodness-of-fit of specifications (A) and (F) based on the BIC suggests that the inclusion of precision dummies leads to a significant improvement in the model's goodness-of-fit. This impression is confirmed on the basis of a LR test, which clearly rejects specification (A) in favor of $(\mathrm{F})$.

In specifications $(G)-(L)$, the precision dummies are interacted with different power functions of $S_{N F}$ of an order up to three. It turns out that orders higher than three are not required and do not significantly improve the model fit. In order to gain sufficient insights into the underlying nonlinear effects, we consider alternative specifications based on different polynomial functions. Specification $(\mathrm{H})$ includes third-order terms 
for low values of the external precision signal, i.e., for $D^{\pi l o w}=1$, and first-order terms for high values of the external precision signal (for specification (I), the reverse is true). Model $(\mathrm{J})$ includes third-order terms for $D^{\pi l o w}=1$ and captures quadratic impacts for $D^{\pi h i g h}=1$ (again, for model $(\mathrm{K})$, the reverse is true). The most comprehensive model (L) includes third-order terms for both low and high values of the external precision signal. However, the LR tests as well as the BIC values favour specification (J). Figure 4 provides a graphical illustration of the estimated price response curves for the best performing specification $(\mathrm{J})$ over the range of observed surprise values.

Finally, a comparison of the models underlying Sections 11.2 and 11.3 is performed on the basis of a LR test of specification (L) against specification (C). Here, specification $(\mathrm{C})$ is clearly rejected. ${ }^{14}$ Note that specifications $(\mathrm{C})$ and $(\mathrm{J})$ yield nearly the same BIC values, which indicates that precision effects do not significantly improve the model's goodness-of-fit over the whole 90-minute period. However, this is due to the fact that price adjustments are mainly observed over the two to four minutes after the announcement, corresponding to $2-4 \%$ of the sample. In this sense, the BIC is not very informative about the statistical (and economic) importance of precision effects. Therefore, we instead rely on the significance of estimates and the employed LR tests which reflect that short-term price adjustments are significantly affected by precision effects.

Thus, we can summarize that both the internal and external precision signals contribute to the explanation of differences in the strength of the price reaction. This suggests that traders try to infer information precision from different sources, not only by looking at the magnitude of the surprise as suggested by Subramanyam (1996), but also by inspecting additional detail information related to the headline figures, as suggested by Hautsch and Hess (2007).

\footnotetext{
${ }^{14}$ Note that model $(\mathrm{C})$ is not nested in $(\mathrm{J})$, and thus a LR test is not applicable.
} 
As shown in Figure 4, prices react in a rather non-linear way if the perceived precision is low. We find strong evidence for an S-shaped price response curve, as predicted by the model in Subramanyam (1996). In particular, the price response coefficient decreases for absolute surprises in the positive as well as negative surprise range. For large, negative surprises, we even obtain some evidence of a negative marginal price reaction.

Moreover, we find evidence that the S-shape of the price response curve is dampened if the external precision signal is high. In that case, the curvature of the price response function significantly declines, and we observe a nearly linear relationship between price changes and surprises; if an announcement figure is perceived to be of high precision, market participants react to big surprises with a strength similar to that of the reaction to small surprises. In contrast, if the external precision measure indicates that the announced information is of low quality, investors react more moderately to larger surprises. Given the nearly linear shape of the price response curve in a state of high information precision, we might be tempted to argue that market participants completely ignore the internal precision signal if the perceived precision is high.

However, the model derived in 11.3 suggests that the opposite is true. In fact, nonlinearities in the price response should be more pronounced for a high value of the external precision signal (recall Figure 1 for an illustration). Within our sample period, we might not have sufficiently large surprise values to observe the situation depicted by Figure 1 (a). Presumably, we face the situation illustrated by Figure 1 (b), where the curvature is dampened in a relatively narrow region around zero and the price response curve becomes almost linear when precision is high. Consequently, we should be careful in interpreting the reduced non-linearities in the price-response curve in periods of high precision.

Note that our results are robust regarding the imposed functional form of the price re- 
sponse relationship. Instead of capturing potential nonlinearities based on power functions, we also estimated the model based on flexible Fourier forms defined over the range of surprises. The fact that we get quantitatively the same results indicates the robustness of our findings. ${ }^{15}$

As can be seen from the following example, our results are also significant from an economic perspective. Assume that market participants observe a median-sized piece of 'good' nonfarm payrolls news (i.e., a nonfarm payrolls figure which is $0.06 \%$ lower than the median forecast) in connection with a 'low' external precision. According to the best performing specification $(\mathrm{J})$ and accounting for both the internal and external precision, prices increase by about $0.31 \%$ in response to this release. If, instead, market participants ignore both precision signals (in accordance with the standard Bayesian learning model in specification (A)), prices would increase by about $0.22 \%$. Hence, ignoring both precision signals would lead to a severe underestimation of the price response by about one third. In contrast, suppose that an extreme surprise of $S_{N F}=-0,18 \%$ is observed, corresponding to the $90 \%$ quantile for 'good news,' again in connection with a 'low' external precision signal. Since the internal signal suggests a very low precision, according to specification $(\mathrm{J})$, prices react only slightly more strongly, i.e., we would observe a return of $0,36 \%$. However, ignoring both precision signals would strongly overestimate price responses by $89 \%$ and result in an expected return of $0,67 \%$.

Overall, these results provide strong evidence in favor of the claim of Bayesian learning that the perceived quality of information plays an important role in determining its price impact. The results suggest that market participants actually use internal as well as external signals to determine the precision of released news. Ignoring the available information on news' precision may result in strong over- or underestimations of the

\footnotetext{
${ }^{15}$ For sake of brevity, the latter results are not included in the paper, but they are available upon request from the authors.
} 
price reaction.

\section{Conclusion}

If agents in financial markets are confronted with new information, they process it by adjusting their expectations on asset values. Bayesian learning provides a concept for processing this information consistently. Since, in contrast to standard assumptions, the precision of information is rarely available to market participants, we derive different settings of Bayesian learning models which allow for uncertainty in the precision of news. Within these models, one common principle remains true: Market participants' perception of information quality plays a major role in determining the strength of price adjustments. However, this perception of precision may be based on different precision signals.

The theoretical models show that the amount of unanticipated information in an announcement may provide traders with an 'internal' signal of its precision, i.e., the price response coefficient is decreasing with the magnitude of surprises. In addition, price responses to news may be influenced by 'external' signals of news precision, such as the reputation of an auditing company, the reliability of a newspaper or the data coverage of an agency. If we observe a high value for such an external precision measure, the price response to a given surprise is relatively stronger than in a situation of low perceived precision.

To test these implications, we focus on the most influential macroeconomic report, i.e., the U.S. employment release. In its headline figures, this report does not contain any release-specific precision measures. Instead, market participants may extract precision measures of the released headline figures by analyzing related detail information. As suggested by Hautsch and Hess (2007), revisions of previously announced figures in 
conjunction with the cross-sectional standard deviations of analysts' forecasts may be used to derive such an external precision measure.

We investigate the price reaction of CBOT T-bond futures to these employment announcements using high-frequency data. The price response curves extracted from the data illustrate a non-linear price impact of information depending on its surprise component. As predicted by the theory, our empirical results suggest that market participants seem to interpret the magnitude of the surprise contained in a news release as an internal indication of its precision. Consequently, if traders observe an announcement that strongly deviates from their expectations, they tend to conclude that this announcement is imprecise.

Using the precision measures proposed by Hautsch and Hess (2007) as an additional external signal of the precision of the released data, we confirm the strong link between the perceived precision of news and the price response. If the precision signal derived from past revision data indicates a high relative precision level of news, market prices react stronger to the unanticipated part of the data. If the external precision signal indicates a poor quality of the released figures, market prices react only weakly.

Overall, our empirical analysis provides evidence in favor of Bayesian learning in cases where the precision of news is uncertain. The results show that the quality of news significantly determines the implied price impact. The results also suggest that if exact quality measures for a release are missing, traders try to infer news' precision by drawing on different sources. When observing a piece of news, they assess for themselves how precise it is. Market participants seem to include in these assessments information on the reliability of the source of the message.

To our knowledge, the present analysis is the first that describes the impact of these two simultaneous - internal and external - precision signals in a unified framework. 
Such Bayesian learning models accounting for uncertain news precision provide further insight into price formation mechanisms and help to assess risky positions. For example, to infer how the release of an unexpectedly high unemployment figure will affect the value of a bond portfolio, traders and portfolio managers need to use an adequate model for the price impact of employment data. We show that ignoring the available information on announcements' precision may result in strong over- or underestimations of the price impact of news. 


\section{Appendix A}

We first derive the posterior beliefs of traders after observing an announcement and a proxy for the precision of the signal. Recall the assumption that the random variables $A$ and $\hat{\rho}_{A}$ are conditionally independent given the precision $\rho_{A}$, i.e.

$$
f_{A, \hat{\rho}_{A} \mid \rho_{A}}\left(A, \hat{\rho}_{A} \mid \rho_{A}\right)=f_{A \mid \rho_{A}}\left(A \mid \rho_{A}\right) f_{\hat{\rho}_{A} \mid \rho_{A}}\left(\hat{\rho}_{A} \mid \rho_{A}\right) .
$$

Then, the conditional expectation of $X$ given $A$ and $\hat{\rho}_{A}$ is given by

$$
\begin{aligned}
\mu_{P} & =E\left[X \mid A, \hat{\rho}_{A}\right] \\
& =E\left[E\left[X \mid A, \hat{\rho}_{A}, \rho_{A}\right] \mid A, \hat{\rho}_{A}\right] \\
& =E\left[\mu_{F}+\left(A-\mu_{F}\right) \rho_{A} / \rho_{F} \mid A, \hat{\rho}_{A}\right] \\
& =\mu_{F}+E\left[\left(A-\mu_{F}\right) \rho_{A} / \rho_{F} \mid A, \hat{\rho}_{A}\right] \\
& =\mu_{F}+\left(A-\mu_{F}\right) E\left[\rho_{A} \mid A, \hat{\rho}_{A}\right] / \rho_{F} \\
& \equiv \mu_{F}+S \cdot \pi\left(S, \hat{\rho}_{A}\right) .
\end{aligned}
$$

The expected precision of the announcement is given as

$$
\begin{aligned}
E\left[\rho_{A} \mid A, \hat{\rho}_{A}\right] & =\int_{S_{A}} \rho_{A} f\left(\rho_{A} \mid A, \hat{\rho}_{A}\right) d \rho_{A} \\
& =\int_{S_{A}} \rho_{A} \frac{f\left(A, \hat{\rho}_{A} \mid \rho_{A}\right) f\left(\rho_{A}\right)}{f\left(A, \hat{\rho}_{A}\right)} d \rho_{A} \\
& =\frac{\int_{S_{A}} \rho_{A} f\left(A \mid \rho_{A}\right) f\left(\hat{\rho}_{A} \mid \rho_{A}\right) f\left(\rho_{A}\right) d \rho_{A}}{f\left(A, \hat{\rho}_{A}\right)} \\
& =\frac{\int_{S_{A}} \rho_{A} f\left(A \mid \rho_{A}\right) f\left(\hat{\rho}_{A} \mid \rho_{A}\right) f\left(\rho_{A}\right) d \rho_{A}}{\int_{S_{A}} f\left(A, \hat{\rho}_{A} \mid \rho_{A}\right) f\left(\rho_{A}\right) d \rho_{A}} \\
& =\frac{\int_{S_{A}} \rho_{A} f\left(A \mid \rho_{A}\right) f\left(\hat{\rho}_{A} \mid \rho_{A}\right) f\left(\rho_{A}\right) d \rho_{A}}{\int_{S_{A}} f\left(A \mid \rho_{A}\right) f\left(\hat{\rho}_{A} \mid \rho_{A}\right) f\left(\rho_{A}\right) d \rho_{A}},
\end{aligned}
$$

where the support of $f\left(\rho_{A}\right)$ is given by $S_{A} \in\left(\rho_{F}, \infty\right)$.

Using these relations we now turn to the proofs of the particular theorems. 
Proof of Theorem 1: Note that $\partial f\left(A \mid \rho_{A}\right) \partial S^{2}=\left(-\frac{1}{2} \rho_{A}\right) f\left(A \mid \rho_{A}\right)$, since we assumed that $A$ is conditionally normally distributed given $\rho_{A}$. We need to show that the partial derivative of the conditional expected precision with respect to the absolute surprise is strictly negative.

$$
\begin{aligned}
\frac{\partial E\left[\rho_{A} \mid A, \hat{\rho}_{A}\right]}{\partial S^{2}}= & \frac{\partial}{\partial S^{2}} \frac{\int_{S_{A}} \rho_{A} f\left(A \mid \rho_{A}\right) f\left(\hat{\rho}_{A} \mid \rho_{A}\right) f\left(\rho_{A}\right) d \rho_{A}}{\int_{S_{A}} f\left(A \mid \rho_{A}\right) f\left(\hat{\rho}_{A} \mid \rho_{A}\right) f\left(\rho_{A}\right) d \rho_{A}} \\
= & \frac{\left(\frac{\partial}{\partial S^{2}} \int_{S_{A}} \rho_{A} f\left(A \mid \rho_{A}\right) f\left(\hat{\rho}_{A} \mid \rho_{A}\right) f\left(\rho_{A}\right) d \rho_{A}\right) \int_{S_{A}} f\left(A \mid \rho_{A}\right) f\left(\hat{\rho}_{A} \mid \rho_{A}\right) f\left(\rho_{A}\right) d \rho_{A}}{\left(\int_{S_{A}} f\left(A \mid \rho_{A}\right) f\left(\hat{\rho}_{A} \mid \rho_{A}\right) f\left(\rho_{A}\right) d \rho_{A}\right)^{2}} \\
& -\frac{\int_{S_{A}} \rho_{A} f\left(A \mid \rho_{A}\right) f\left(\hat{\rho}_{A} \mid \rho_{A}\right) f\left(\rho_{A}\right) d \rho_{A}\left(\frac{\partial}{\partial S^{2}} \int_{S_{A}} f\left(A \mid \rho_{A}\right) f\left(\hat{\rho}_{A} \mid \rho_{A}\right) f\left(\rho_{A}\right) d \rho_{A}\right)}{\left(\int_{S_{A}} f\left(A \mid \rho_{A}\right) f\left(\hat{\rho}_{A} \mid \rho_{A}\right) f\left(\rho_{A}\right) d \rho_{A}\right)^{2}} \\
= & \frac{-\frac{1}{2} \int_{S_{A}} \rho_{A}^{2} f\left(A \mid \rho_{A}\right) f\left(\hat{\rho}_{A} \mid \rho_{A}\right) f\left(\rho_{A}\right) d \rho_{A} \int_{S_{A}} f\left(A \mid \rho_{A}\right) f\left(\hat{\rho}_{A} \mid \rho_{A}\right) f\left(\rho_{A}\right) d \rho_{A}}{\left(\int_{S_{A}} f\left(A \mid \rho_{A}\right) f\left(\hat{\rho}_{A} \mid \rho_{A}\right) f\left(\rho_{A}\right) d \rho_{A}\right)^{2}} \\
= & -\frac{\int_{S_{A}} \rho_{A} f\left(A \mid \rho_{A}\right) f\left(\hat{\rho}_{A} \mid \rho_{A}\right) f\left(\rho_{A}\right) d \rho_{A}\left(-\frac{1}{2}\right) \int_{S_{A}} \rho_{A} f\left(A \mid \rho_{A}\right) f\left(\hat{\rho}_{A} \mid \rho_{A}\right) f\left(\rho_{A}\right) d \rho_{A}}{2 \int_{S_{A}} f\left(A \mid \rho_{A}\right) f\left(\hat{\rho}_{A} \mid \rho_{A}\right) f\left(\rho_{A}\right) d \rho_{A}} \\
& +\frac{\left(\int_{S_{A}} \rho_{A} f\left(A \mid \rho_{A}\right) f\left(\hat{\rho}_{A} \mid \rho_{A}\right) f\left(\rho_{A}\right) d \rho_{A}\right)^{2}}{2\left(\int_{S_{A}} f\left(A \mid \rho_{A}\right) f\left(\hat{\rho}_{A} \mid \rho_{A}\right) f\left(\rho_{A}\right) d \rho_{A}\right)^{2}} \\
= & -\frac{1}{2}\left[\frac{\int_{S_{A}} \rho_{A}^{2} f\left(A \mid \rho_{A}\right) f\left(\hat{\rho}_{A} \mid \rho_{A}\right) f\left(\rho_{A}\right) d \rho_{A}}{\int_{S_{A}} f\left(A \mid \rho_{A}\right) f\left(\hat{\rho}_{A} \mid \rho_{A}\right) f\left(\rho_{A}\right) d \rho_{A}}\right. \\
& \left.-\left(\frac{\int_{S_{A}} \rho_{A} f\left(A \mid \rho_{A}\right) f\left(\hat{\rho}_{A} \mid \rho_{A}\right) f\left(\rho_{A}\right) d \rho_{A}}{\int_{S_{A}} f\left(A \mid \rho_{A}\right) f\left(\hat{\rho}_{A} \mid \rho_{A}\right) f\left(\rho_{A}\right) d \rho_{A}}\right)^{2}\right] \\
= & -\frac{1}{2}\left(E\left[\rho_{A}^{2} \mid A, \hat{\rho}_{A}\right]-\left(E\left[\rho_{A} \mid A, \hat{\rho}_{A}\right]\right)^{2}\right) \\
= & -\frac{1}{2} \operatorname{Var}\left[\rho_{A} \mid A, \hat{\rho}_{A}\right]<0,
\end{aligned}
$$

for any non-degenerate distribution of the precision $\rho_{A}$. Since $|S|$ and $S^{2}$ are positively and monotonically related, the result can be applied for $|S|$. Then, it is straightforwardly shown that $\partial \pi\left(S, \hat{\rho}_{A}\right) / \partial|S|<0$. 
Proof of Theorem 2: Note that $\partial f\left(A \mid \rho_{A}\right) \partial S=-\rho_{A} S f\left(A \mid \rho_{A}\right)$, since we assume that $A$ is conditionally normally distributed given $\rho_{A}$. Then,

$$
\frac{\partial E\left[\rho_{A} \mid A, \hat{\rho}_{A}\right]}{\partial S}=2 S \cdot \frac{\partial E\left[\rho_{A} \mid A, \hat{\rho}_{A}\right]}{\partial S^{2}} .
$$

Hence, using Theorem 1 we get

$$
\frac{\partial E\left[\rho_{A} \mid A, \hat{\rho}_{A}\right]}{\partial S}=-S \cdot \operatorname{Var}\left[\rho_{A} \mid A, \hat{\rho}_{A}\right]
$$

and thus

$$
\frac{\partial\left(\mu_{P}-\mu_{F}\right)}{\partial S}=\pi\left(S, \hat{\rho}_{A}\right)+\frac{S}{\rho_{F}} \frac{\partial E\left[\rho_{A} \mid A, \hat{\rho}_{A}\right]}{\partial S}=\pi\left(S, \hat{\rho}_{A}\right)-\frac{S^{2}}{\rho_{F}} \operatorname{Var}\left[\rho_{A} \mid A, \hat{\rho}_{A}\right] .
$$


Proof of Theorem 3: Note that $\partial f\left(\hat{\rho}_{A} \mid \rho_{A}\right) \partial \hat{\rho}_{A}=\left(-\frac{\hat{\rho}_{A}-\rho_{A}}{2 \sigma_{\hat{\rho}_{A}}^{2}}\right) f\left(\hat{\rho}_{A} \mid \rho_{A}\right)$, since we assume a normal distribution for $\hat{\rho}_{A}$. We need to show that the partial derivative of the conditional expected precision with respect to the precision signal is strictly positive,

$$
\begin{aligned}
& \frac{\partial E\left[\rho_{A} \mid A, \hat{\rho}_{A}\right]}{\partial \hat{\rho}_{A}} \\
= & \frac{\int_{S_{A}} \rho_{A} f\left(A \mid \rho_{A}\right) \frac{\partial f\left(\hat{\rho}_{A} \mid \rho_{A}\right)}{\partial \hat{\rho}_{A}} f\left(\rho_{A}\right) d \rho_{A} \int_{S_{A}} f\left(A \mid \rho_{A}\right) f\left(\hat{\rho}_{A} \mid \rho_{A}\right) f\left(\rho_{A}\right) d \rho_{A}}{\left(\int_{S_{A}} f\left(A \mid \rho_{A}\right) f\left(\hat{\rho}_{A} \mid \rho_{A}\right) f\left(\rho_{A}\right) d \rho_{A}\right)^{2}} \\
- & \frac{\int_{S_{A}} \rho_{A} f\left(A \mid \rho_{A}\right) f\left(\hat{\rho}_{A} \mid \rho_{A}\right) f\left(\rho_{A}\right) d \rho_{A} \int_{S_{A}} f\left(A \mid \rho_{A}\right) \frac{\partial f\left(\hat{\rho}_{A} \mid \rho_{A}\right)}{\partial \hat{\rho}_{A}} f\left(\rho_{A}\right) d \rho_{A}}{\left(\int_{S_{A}} f\left(A \mid \rho_{A}\right) f\left(\hat{\rho}_{A} \mid \rho_{A}\right) f\left(\rho_{A}\right) d \rho_{A}\right)^{2}} \\
= & \frac{\int_{S_{A}} \rho_{A} f\left(A \mid \rho_{A}\right)\left(-\frac{\hat{\rho}_{A}-\rho_{A}}{2 \sigma_{\hat{\rho}_{A}}^{2}}\right) f\left(\hat{\rho}_{A} \mid \rho_{A}\right) f\left(\rho_{A}\right) d \rho_{A} \int_{S_{A}} f\left(A \mid \rho_{A}\right) f\left(\hat{\rho}_{A} \mid \rho_{A}\right) f\left(\rho_{A}\right) d \rho_{A}}{\left(\int_{S_{A}} f\left(A \mid \rho_{A}\right) f\left(\hat{\rho}_{A} \mid \rho_{A}\right) f\left(\rho_{A}\right) d \rho_{A}\right)^{2}} \\
- & \frac{\int_{S_{A}} \rho_{A} f\left(A \mid \rho_{A}\right) f\left(\hat{\rho}_{A} \mid \rho_{A}\right) f\left(\rho_{A}\right) d \rho_{A} \int_{S_{A}} f\left(A \mid \rho_{A}\right)\left(-\frac{\hat{\rho}_{A}-\rho_{A}}{2 \sigma_{\hat{\rho}_{A}}^{2}}\right) f\left(\hat{\rho}_{A} \mid \rho_{A}\right) f\left(\rho_{A}\right) d \rho_{A}}{\left.\left(A \mid \rho_{A}\right) f\left(\hat{\rho}_{A} \mid \rho_{A}\right) f\left(\rho_{A}\right) d \rho_{A}\right)^{2}} \\
= & \frac{1}{2 \sigma_{\hat{\rho}_{A}}^{2}}\left[\frac{\int_{S_{A}} \rho_{A}^{2} f\left(A \mid \rho_{A}\right) f\left(\hat{\rho}_{A} \mid \rho_{A}\right) f\left(\rho_{A}\right) d \rho_{A}}{\int_{S_{A}} f\left(A \mid \rho_{A}\right) f\left(\hat{\rho}_{A} \mid \rho_{A}\right) f\left(\rho_{A}\right) d \rho_{A}}\right. \\
- & \left.\left(\frac{\int_{S_{A}} \rho_{A} f\left(A \mid \rho_{A}\right) f\left(\hat{\rho}_{A} \mid \rho_{A}\right) f\left(\rho_{A}\right) d \rho_{A}}{\int_{S_{A}} f\left(A \mid \rho_{A}\right) f\left(\hat{\rho}_{A} \mid \rho_{A}\right) f\left(\rho_{A}\right) d \rho_{A}}\right)^{2}\right] \\
= & \frac{1}{2 \sigma_{\hat{\rho}_{A}}^{2}}\left(E\left[\rho_{A}^{2} \mid A, \hat{\rho}_{A}\right]-\left(E\left[\rho_{A} \mid A, \hat{\rho}_{A}\right]\right)^{2}\right) \\
= & \frac{1}{2 \sigma_{\hat{\rho}_{A}}^{2}} \operatorname{Var}\left[\rho_{A} \mid A, \hat{\rho}_{A}\right]>0,
\end{aligned}
$$

for any non-degenerate distribution of the precision $\rho_{A}$. Then, it is straightforwardly shown that $\partial \pi\left(S, \hat{\rho}_{A}\right) / \partial \hat{\rho}_{A}>0$ and $\partial\left|\mu_{P}-\mu_{F}\right| / \partial \hat{\rho}_{A}>0$. 
Proof of Theorem 4: The posterior mean of $X$ is written as

$$
\begin{aligned}
\mu_{P} & =E\left[X \mid A, \hat{\rho}_{A}\right] \\
& =E\left[E\left[X \mid A, \rho_{F}, \rho_{A}, \hat{\rho}_{A}\right] \mid A, \hat{\rho}_{A}\right] \\
& =E\left[\left(A-\mu_{F}\right) \rho_{A} / \rho_{F}+\mu_{F} \mid A, \hat{\rho}_{A}\right] \\
& =\mu_{F}+\left(A-\mu_{F}\right) \cdot E\left[\rho_{A} / \rho_{F} \mid A, \hat{\rho}_{A}\right] \\
& =\mu_{F}+\left(A-\mu_{F}\right) \cdot \int_{S_{F}} \int_{S_{A}} \rho_{A} / \rho_{F} f\left(A, \hat{\rho}_{A} \mid \rho_{A}, \rho_{F}\right) f\left(\rho_{A}, \rho_{F}\right) d \rho_{A} d \rho_{F} \\
& =\mu_{F}+\left(A-\mu_{F}\right) \cdot \int_{S_{F}} 1 / \rho_{F} \underbrace{\int_{S_{A}} \rho_{A} f\left(A, \hat{\rho}_{A} \mid \rho_{A}, \rho_{F}\right) f\left(\rho_{A} \mid \rho_{F}\right) d \rho_{A}}_{E\left[\rho_{A} \mid A, \rho_{F}, \hat{\rho}_{A}\right]} f\left(\rho_{F}\right) d \rho_{F} \\
& \equiv \mu_{F}+\left(A-\mu_{F}\right) \cdot \pi\left(S, \hat{\rho}_{A}\right) .
\end{aligned}
$$

Using the result established in Theoreom 1, we can show

$$
\begin{aligned}
\partial \pi(S) / \partial S^{2} & =\frac{\partial}{\partial S^{2}} \int_{S_{F}} 1 / \rho_{F} \int_{S_{A}} \rho_{A} f\left(A, \hat{\rho}_{A} \mid \rho_{A}, \rho_{F}\right) f\left(\rho_{A} \mid \rho_{F}\right) d \rho_{A} f\left(\rho_{F}\right) d \rho_{F} \\
& =\int_{S_{F}} 1 / \rho_{F} \frac{\partial \int_{S_{A}} \rho_{A} f\left(A, \hat{\rho}_{A} \mid \rho_{A}, \rho_{F}\right) f\left(\rho_{A} \mid \rho_{F}\right) d \rho_{A}}{\partial S^{2}} f\left(\rho_{F}\right) d \rho_{F} \\
& =\int_{S_{F}} 1 / \rho_{F} \underbrace{\frac{\partial E\left[\rho_{A} \mid A, \rho_{F}, \hat{\rho}_{A}\right]}{\partial\left(S^{2}\right)}}_{<0} f\left(\rho_{F}\right) d \rho_{F}<0 .
\end{aligned}
$$

Here, the price response coefficient $\pi\left(S, \hat{\rho}_{A}\right)$ is just a weighted average of the price response coefficients in the case of known variance of the prior information weighted by the corresponding probability. Hence, the results established by Theorems 1 and 3 still hold. 


\section{Appendix B}

FIGURE 1:

Price response curves resulting from the Bayesian learning model

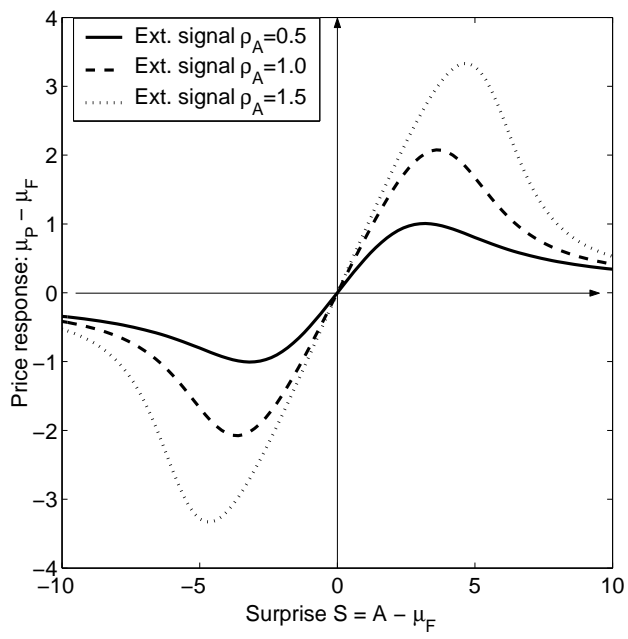

(a) Large range of surprises

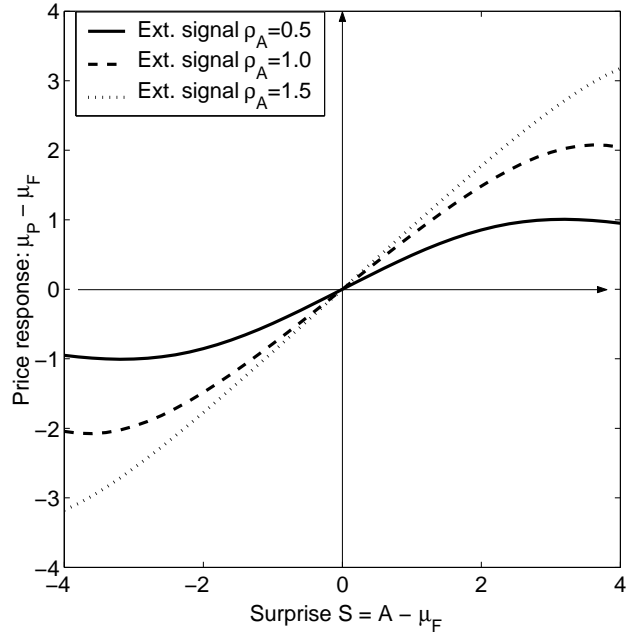

(b) Small range of surprises

x-axis: surprises in the announcement $S=\left(A-\mu_{A}\right)$, i.e. deviations of the announced figure from its mean, y-axis: price responses, i.e. changes in expectations $\mu_{P}-\mu_{F}$. The graphs show a numerical example of price response curves given the model specification in section 11.3. Prior beliefs are normally distributed with $\mu_{F}=0$ and $\rho_{F}=1$, while news' precision $\rho_{A}$ follows a truncated gamma distribution with scale parameter $\lambda=1$ and shape parameter $r=1$. Additionally, an external estimate of news' precision $\hat{\rho}_{A}$ is observed which is normally distributed as $\hat{\rho}_{A} \sim N\left(\rho_{A}, \sigma_{\hat{\rho}_{A}}\right)$. Price response curves are increasing in the observed value of the precision proxy, the graphs correspond to external precision signals of $\hat{\rho}_{A}=0.5,1,1.5$ while we choose $\sigma_{\hat{\rho}_{A}}=0.25$. 
FIGURE 2:

Estimated price-response curve allowing for internal precision signals

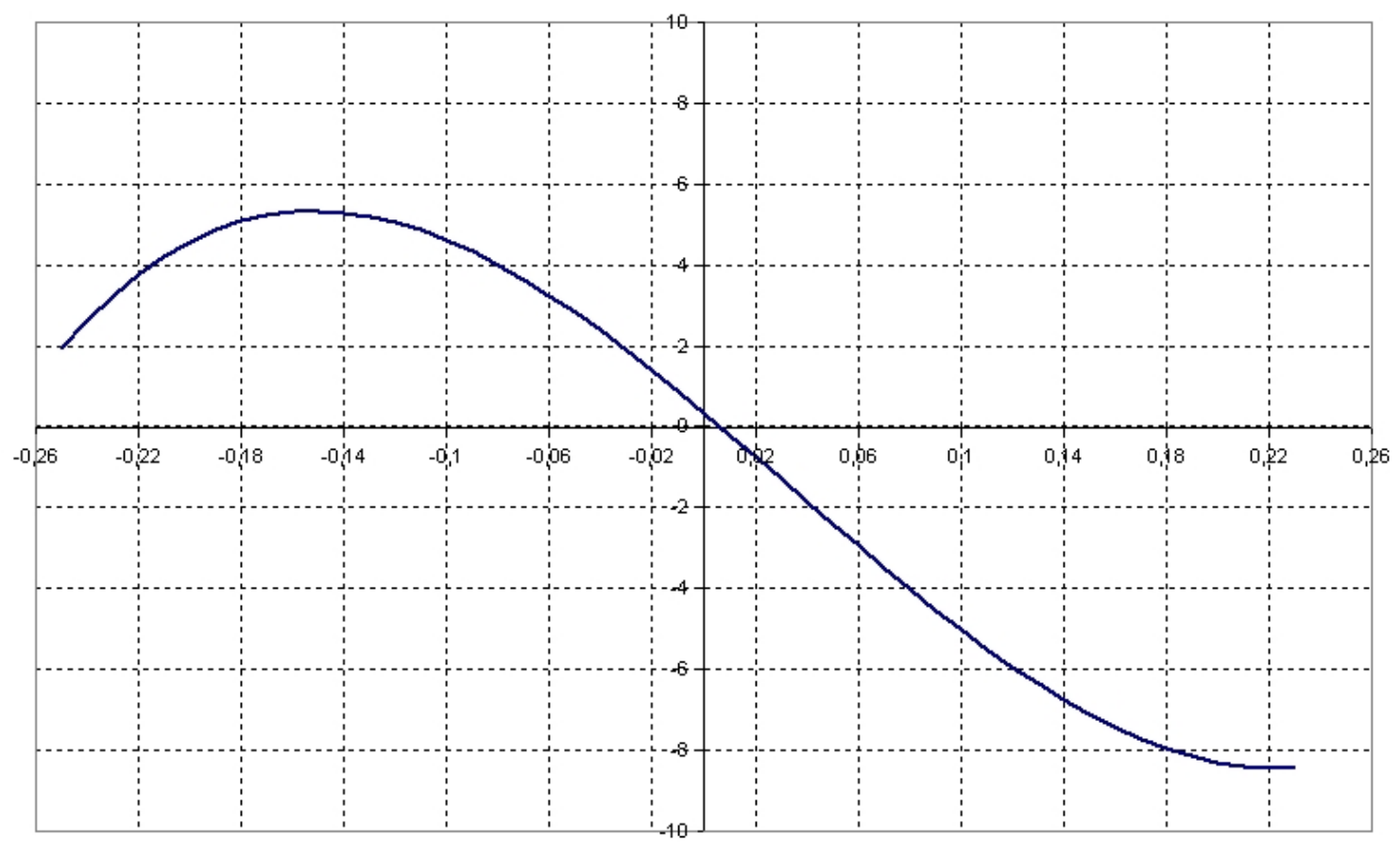

x-axis: surprises in the U.S. nonfarm payrolls figure $S_{N F}$ (in percentage points), y-axis: estimated price response $\hat{r_{t}}(\log$-returns $\times 1000)$. This figure provides an illustration of the price response curve to surprises in announcements of nonfarm payrolls figures corresponding to specification $(\mathrm{C})$ in table 1 . The results are based on a QML estimation of $\mathrm{AR}(2)-\mathrm{ARCH}(3)$ models for two-minute log returns during the intraday interval 8:22-9:52 a.m. EST on employment announcement days on which no other macroeconomic report was released at the same time. The sample period is Jan. 1991 - Dec. 2005, resulting in 7245 observations (i.e., 161 days with no overlapping announcements $\times 45$ two-min intervals). According to the Bayes information criterion (BIC), the model that includes polynomial terms in nonfarm payrolls surprises up to the third order provides the best specification. Thus, as predicted by the theoretical model, the resulting price response curves are non-linear, since big surprises serve as a signal for low news' precision. Therefore, big surprises in the announced figure lead to relatively weaker price reactions than small surprises do. 
FIGURE 3:

Estimated linear price-response curves for high and low external precision signals

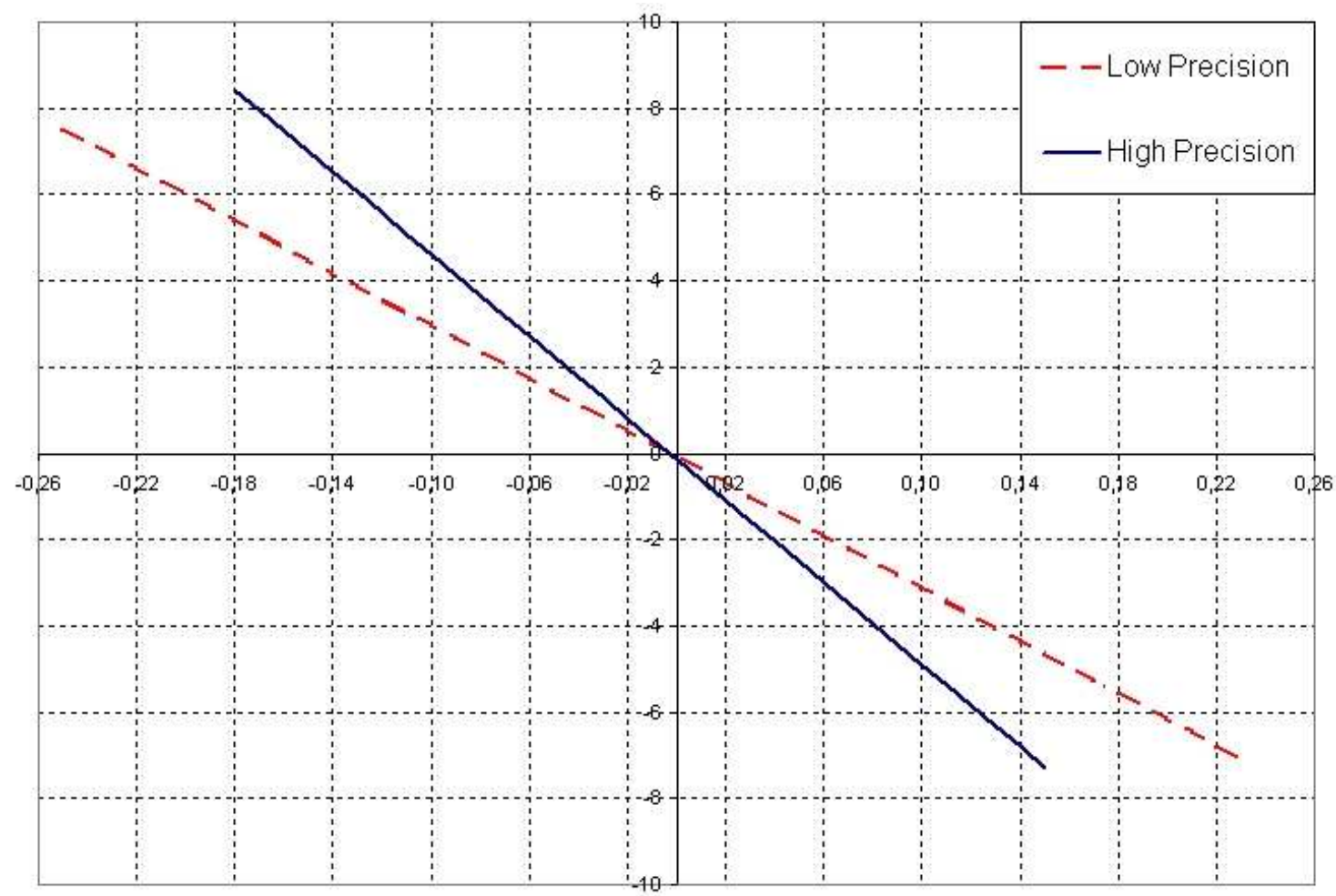

x-axis: surprises in the U.S. nonfarm payrolls figure $S_{N F}$ (in percentage points), y-axis: estimated price response $\hat{r_{t}}$ (log-returns $\left.\times 1000\right)$. A graphical illustration of the price response curve to nonfarm payrolls figures as described by specification (F) in table 2. The results are based on a QML estimation of AR(2)$\mathrm{ARCH}(3)$ models for two-min log returns during the intraday interval 8:22 - 9:52 a.m. EST on employment announcement days for which no other macroeconomic report is released at the same time. The sample period is Jan. 1991 - Dec. 2005, resulting in 7245 observations (i.e., 161 days with no overlapping announcements $\times 45$ two-min intervals). The curve corresponding to high precision signals has a significantly larger slope. A higher external precision signal leads to stronger price reactions given the same amount of unexpected information in a news release. 
FIGURE 4:

Estimated price-response curves allowing for internal and external precision signals

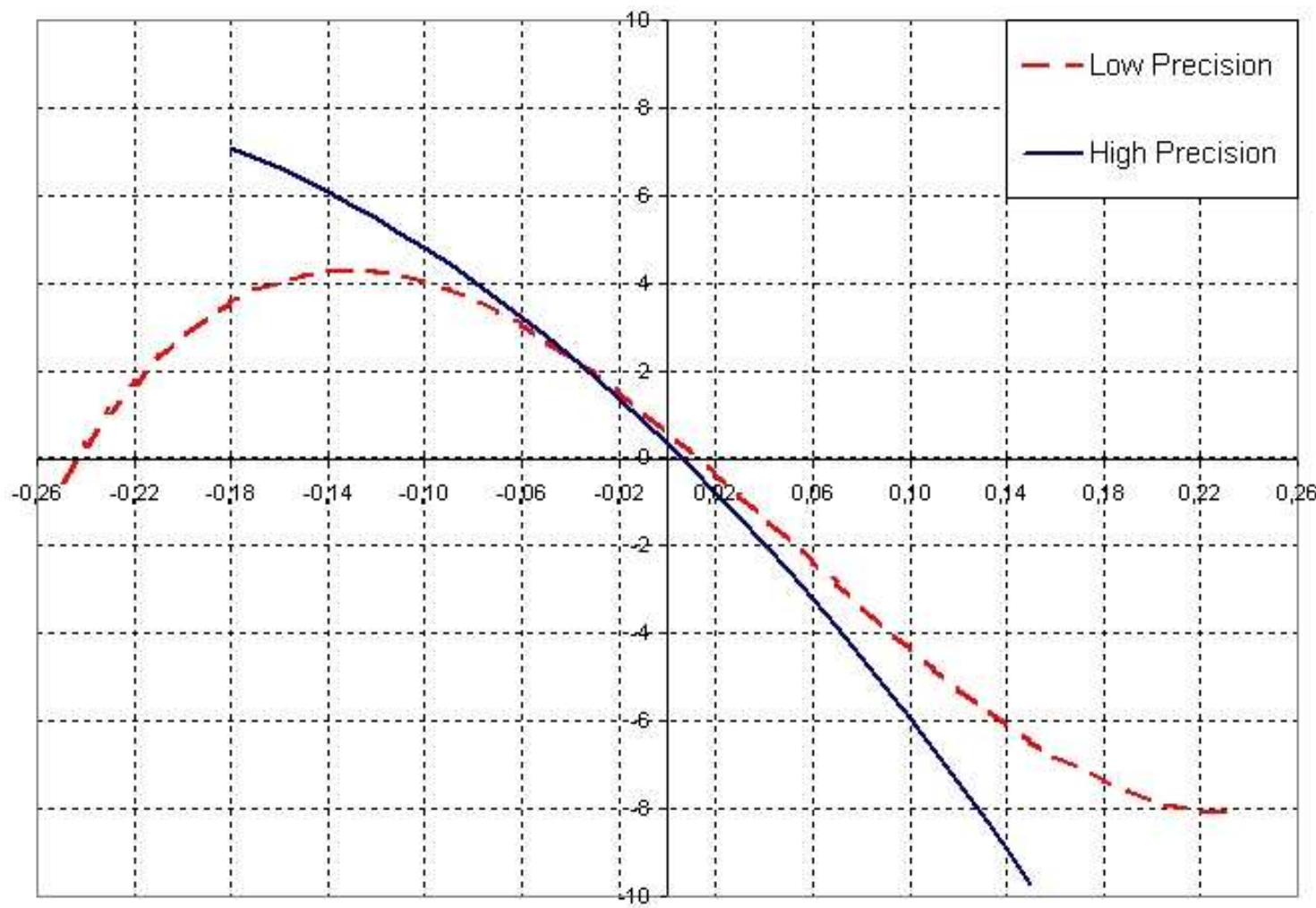

x-axis: surprises in the U.S. nonfarm payrolls figure $S_{N F}$ (in percentage points), y-axis: estimated price response $\hat{r_{t}}(\log$-returns $\times 1000)$. A graphical illustration of the price response curve to nonfarm payrolls figures as described by specification $(\mathrm{J})$ in table 2 . The results are based on a QML estimation of $\operatorname{AR}(2)-$ $\mathrm{ARCH}(3)$ models for two-min log returns during the intraday interval 8:22-9:52 a.m. EST on employment announcement days on which no other macroeconomic report was released at the same time. The sample period is Jan. 1991 - Dec. 2005, resulting in 7245 observations (i.e., 161 days with no overlapping announcements $\times 45$ two-min intervals). Prices tend to react more strongly to news with a high precision signal. For high precision signals, polynomial terms in nonfarm payroll surprises are captured only up to the second order, while for low precision signals, terms up to the third order are included. 
TABLE 1

Estimation of price response functions with surprises as an internal precision signal

\begin{tabular}{|c|c|c|c|c|c|}
\hline Model & (A) & (B) & (C) & (D) & (E) \\
\hline \multicolumn{6}{|l|}{ Mean equation } \\
\hline cons & $-0,002$ & $-0,002$ & $-0,002$ & $-0,002$ & $-0,002$ \\
\hline$D^{8: 28} \cdot S_{N F}$ & 4,406 & 3,619 & 3,927 & 4,078 & 3,829 \\
\hline$D^{8: 30}$ & $-0,080$ & 0,530 & 0,355 & 0,558 & 0,637 \\
\hline$D^{8: 30} \cdot S_{N F}^{1}$ & $-37,873^{* * *}$ & $-42,415 * * *$ & $-53,447 * * *$ & $-54,909 * * *$ & $-50,344 * * *$ \\
\hline$D^{8: 30} \cdot S_{N F}^{2}$ & & $-91,220 * *$ & $-55,336 *$ & $-119,526$ & $-145,530$ \\
\hline$D^{8: 30} \cdot S_{N F}^{3}$ & & & $531,752 * * *$ & $624,49 * * *$ & 80,073 \\
\hline$D^{8: 30} \cdot S_{N F}^{4}$ & & & & 1658,397 & 2337,978 \\
\hline$D^{8: 30} \cdot S_{N F}^{5}$ & & & & & 9945,756 \\
\hline$D^{8: 32} \cdot S_{N F}$ & $-4,000 * *$ & $-4,322 * *$ & $-4,274^{* *}$ & $-4,181 * *$ & $-4,277^{* *}$ \\
\hline$D^{8: 28} \cdot S_{U N}$ & 1,636 & 1,278 & 1,320 & 1,314 & 1,175 \\
\hline$D^{8: 30} \cdot S_{U N}$ & $5,003 * *$ & $5,617 * *$ & $5,746 * * *$ & $6,212 * * *$ & $6,367 * * *$ \\
\hline$D^{8: 32} \cdot S_{U N}$ & $1,448 *$ & 1,325 & 1,356 & 1,332 & 1,286 \\
\hline$D^{8: 28} \cdot R_{N F}$ & 2,206 & 1,841 & 2,010 & 1,999 & 1,839 \\
\hline$D^{8: 30} \cdot R_{N F}$ & $-6,872 * * *$ & $-6,390 * * *$ & $-6,215 * *$ & $-5,889 * *$ & $-5,808 * *$ \\
\hline$D^{8: 32} \cdot R_{N F}$ & 0,083 & $-0,428$ & $-0,071$ & $-0,106$ & $-0,258$ \\
\hline$r_{t-1}$ & $-0,091 * * *$ & $-0,091 * * *$ & $-0,090 * * *$ & $-0,090 * * *$ & $-0,091 * * *$ \\
\hline$r_{t-2}$ & $-0,001$ & 0,000 & 0,000 & 0,000 & 0,000 \\
\hline \multicolumn{6}{|l|}{ Variance equation } \\
\hline cons & $0,439 * * *$ & $0,439 * * *$ & $0,436 * * *$ & $0,436 * * *$ & $0,437^{* * *}$ \\
\hline$A R C H(1)$ & $0,148 * *$ & $0,141 * * *$ & $0,146 * * *$ & $0,145 * * *$ & $0,144^{* * *}$ \\
\hline$A R C H(2)$ & $0,057 * * *$ & $0,059 * * *$ & $0,058 * * *$ & $0,058 * * *$ & $0,058 * * *$ \\
\hline$A R C H(3)$ & $0,031 * * *$ & $0,033 * * *$ & $0,034 * * *$ & $0,034 * * *$ & $0,034 * * *$ \\
\hline LL & $-8020,69$ & $-7999,57$ & $-7987,29$ & $-7985,32$ & $-7984,12$ \\
\hline $\mathrm{BIC}$ & 2,2485 & 2,2439 & 2,2417 & 2,2424 & 2,2433 \\
\hline LR-Test against model (A) & & $42,24 * * *$ & $66,80 * * *$ & $70,73 * * *$ & $73,13^{* * *}$ \\
\hline LR-Test against model (B) & & & $24,56 * * *$ & $28,49 * * *$ & $30,89 * * *$ \\
\hline LR-Test against model (C) & & & & $3,93 * *$ & $6,33 * *$ \\
\hline LR-Test against model (D) & & & & & 2,40 \\
\hline
\end{tabular}


TABLE 1 (continued)

QML estimation of $\mathrm{AR}(2)-\mathrm{ARCH}(3)$ models for two-min log returns during the intraday interval 8:22-9:52 a.m. EST on employment announcement days for which no other macroeconomic report is released at the same time. The sample period is Jan. 1991 - Dec. 2005, resulting in 7245 observations (i.e. 161 days with no overlapping announcements $\times 452$-min intervals).

The estimated model for log returns $r_{t}$ is given by $r_{t}=c+\sum_{j=1}^{2} \phi_{j} r_{t-j}+x_{t}^{\prime} \beta+\varepsilon_{t}$, where $\varepsilon_{t} \sim N\left(0, h_{t}\right)$, $t$ indexes the first interval after the announcement, 8:30-8:32 a.m., $x_{t}$ denotes a vector of explanatory variables and $\beta$ is the corresponding coefficient vector. $h_{t}$ is given by $h_{t}=\omega+\sum_{j=1}^{3} \psi_{j} \varepsilon_{t-j}^{2}+s_{t}$, where $s_{t}=\delta^{s} \cdot \bar{t}+\sum_{j=1}^{5}\left(\delta_{c, j}^{s} \cos (j \cdot \bar{t} \cdot 2 \pi)+\delta_{s, j}^{s} \sin (j \cdot \bar{t} \cdot 2 \pi)\right)$ denotes the seasonality function based on the parameters $\delta^{s}, \delta_{c, j}^{s}, \delta_{s, j}^{s}$ and a normalized time trend $\bar{t} \in[0,1]$ given by the elapsed time (in minutes) in the interval 8:22 to 9:52 a.m. divided by 90 . The estimated seasonality parameters are omitted in the table.

The regressors $x_{t}$ are the surprise in U.S. nonfarm payrolls, $S_{N F}$, and in unemployment rates, $S_{U N}$, as well as revisions of nonfarm payrolls $R_{N F}$ interacted with time dummies indicating the intervals 8:28-8:30 a.m. $\left(D^{8: 28}\right)$, 8:30-8:32 a.m. $\left(D^{8: 30}\right)$ and 8:32-8:34 a.m. $\left(D^{8: 32}\right)$. To capture non-linear immediate price responses in the interval 8:30-8:32, surprises in nonfarm payrolls $S_{N F}$ are included as polynomials up to the order 5. Surprises are computed based on U.S. employment report figures released by the BLS and consensus forecasts provided by Informa Global Markets, formerly MMS.

The table reports the log likelihood (LL), the Bayes information criterion (BIC) and $\chi^{2}$ statistics of LR tests on the inequality of individual parameters. Statistical inference is based on QML standard errors (Bollerslev and Wooldridge 1992). ${ }^{* * *},{ }^{* *}$, and ${ }^{*}$ indicates significance at the $1 \%, 5 \%$, and $10 \%$ level, respectively. Except for the LR tests, the level of significance is based on two-sided tests. 
TABLE 2

Estimation of price response functions differentiated by low and high values of the additional external precision proxy

\begin{tabular}{|c|c|c|c|c|}
\hline Model & $(\mathrm{F})$ & (G) & $(\mathbf{H})$ & (I) \\
\hline \multicolumn{5}{|l|}{ Mean equation } \\
\hline cons & $-0,002$ & $-0,002$ & $-0,002$ & $-0,002$ \\
\hline$D^{8: 28} \cdot S_{N F}$ & 4,365 & 3,411 & 3,862 & 4,147 \\
\hline$D^{8: 30} \cdot D^{\text {slow }}$ & $-0,074$ & 0,782 & 0,611 & $-0,069$ \\
\hline$D^{8: 30} \cdot D^{\pi h i g h}$ & $-0,141$ & 0,315 & $-0,131$ & 0,203 \\
\hline$D^{8: 30} \cdot S_{N F}^{1} \cdot D^{\pi l o w}$ & $-30,439 * * *$ & $-34,498 * * *$ & $-46,901 * * *$ & $-30,356 * * *$ \\
\hline$D^{8: 30} \cdot S_{N F}^{1} \cdot D^{\pi h i g h}$ & $-47,601 * * *$ & $-53,413 * * *$ & $-47,713 * * *$ & $-56,190 * * *$ \\
\hline$D^{8: 30} \cdot S_{N F}^{2} \cdot D^{\pi l o w}$ & & $-106,693 * *$ & $-77,937 * *$ & \\
\hline$D^{8: 30} \cdot S_{N F}^{2} \cdot D^{\pi h i g h}$ & & $-87,544 *$ & & $-61,651$ \\
\hline$D^{8: 30} \cdot S_{N F}^{3} \cdot D^{\pi l o w}$ & & & $503,544 * *$ & \\
\hline$D^{8: 30} \cdot S_{N F}^{3} \cdot D^{\pi h i g h}$ & & & & 217,349 \\
\hline$D^{8: 32} \cdot S_{N F}$ & $-4,020 * *$ & $-4,694^{* *}$ & $-4,422 * *$ & $-4,305 * *$ \\
\hline$D^{8: 28} \cdot S_{U N}$ & 1,623 & 1,197 & 1,327 & 1,499 \\
\hline$D^{8: 30} \cdot S_{U N}$ & $5,553 * *$ & $6,275 * * *$ & $6,286 * * *$ & $5,723 * *$ \\
\hline$D^{8: 32} \cdot S_{U N}$ & $1,484 *$ & 1,286 & 1,414 & 1,349 \\
\hline$D^{8: 28} \cdot R_{N F}$ & 2,051 & 1,701 & 1,989 & 1,885 \\
\hline$D^{8: 30} \cdot R_{N F}$ & $-5,901 * *$ & $-5,220 * *$ & $-5,424 * *$ & $-5,585 * *$ \\
\hline$D^{8: 32} \cdot R_{N F}$ & $-0,080$ & $-0,664$ & 0,010 & $-0,460$ \\
\hline$r_{t-1}$ & $-0,091 * * *$ & $-0,091 * * *$ & $-0,091 * * *$ & $-0,090 * * *$ \\
\hline$r_{t-2}$ & 0,000 & 0,000 & 0,000 & 0,000 \\
\hline \multicolumn{5}{|l|}{ Variance equation } \\
\hline cons & $0,437 * * *$ & $0,437 * * *$ & $0,437 * * *$ & $0,437 * * *$ \\
\hline$A R C H(1)$ & $0,151^{* *}$ & $0,143 * *$ & $0,148 * *$ & $0,148 * *$ \\
\hline$A R C H(2)$ & $0,057 * * *$ & $0,059 * * *$ & $0,057 * * *$ & $0,058 * * *$ \\
\hline$A R C H(3)$ & $0,032 * * *$ & $0,035 * * *$ & $0,033 * * *$ & $0,034 * * *$ \\
\hline $\mathrm{LL}$ & $-8008,54$ & $-7982,99$ & $-7981,52$ & $-8002,00$ \\
\hline $\mathrm{BIC}$ & 2,2476 & 2,2430 & 2,2426 & 2,2482 \\
\hline LR-Test against model (A) & $24,30^{* * *}$ & $75,40 * * *$ & $78,34^{* * *}$ & $37,38^{* * *}$ \\
\hline \multicolumn{5}{|l|}{ LR-Test against model (C) } \\
\hline LR-Test against model (F) & & $51,10^{* * *}$ & $54,04 * * *$ & $13,08^{* * *}$ \\
\hline
\end{tabular}


TABLE 2 (continued)

Estimation of price response functions differentiated by low and high values of the additional external precision proxy

\begin{tabular}{|c|c|c|c|}
\hline Model & $(\mathbf{J})$ & $(\mathbf{K})$ & (L) \\
\hline \multicolumn{4}{|l|}{ Mean equation } \\
\hline cons & $-0,002$ & $-0,002$ & $-0,002$ \\
\hline$D^{8: 28} \cdot S_{N F}$ & 3,660 & 3,400 & 3,651 \\
\hline$D^{8: 30} \cdot D^{\pi l o w}$ & 0,630 & 0,774 & 0,624 \\
\hline$D^{8: 30} \cdot D^{\pi h i g h}$ & 0,323 & 0,236 & 0,264 \\
\hline$D^{8: 30} \cdot S_{N F}^{1} \cdot D^{\text {nlow }}$ & $-46,957 * * *$ & $-34,580 * * *$ & $-46,950 * * *$ \\
\hline$D^{8: 30} \cdot S_{N F}^{1} \cdot D^{\pi h i g h}$ & $-53,605 * * *$ & $-55,948 * * *$ & $-55,534 * * *$ \\
\hline$D^{8: 30} \cdot S_{N F}^{2} \cdot D^{\pi l o w}$ & $-78,320 * *$ & $-106,453 * *$ & $-78,297 * *$ \\
\hline$D^{8: 30} \cdot S_{N F}^{2} \cdot D^{\pi h i g h}$ & $-89,156^{*}$ & $-66,847$ & $-73,585$ \\
\hline$D^{8: 30} \cdot S_{N F}^{3} \cdot D^{\pi l o w}$ & $512,233 * *$ & & $509,326 * *$ \\
\hline$D^{8: 30} \cdot S_{N F}^{3} \cdot D^{\pi h i g h}$ & & 186,343 & 141,106 \\
\hline$D^{8: 32} \cdot S_{N F}$ & $-4,692 * *$ & $-4,693 * *$ & $-4,692 * *$ \\
\hline$D^{8: 28} \cdot S_{U N}$ & 1,169 & 1,207 & 1,176 \\
\hline$D^{8: 30} \cdot S_{U N}$ & $6,694 * * *$ & $6,097 * * *$ & $6,556 * * *$ \\
\hline$D^{8: 32} \cdot S_{U N}$ & 1,230 & 1,338 & 1,267 \\
\hline$D^{8: 28} \cdot R_{N F}$ & 1,707 & 1,745 & 1,744 \\
\hline$D^{8: 30} \cdot R_{N F}$ & $-4,736 * *$ & $-5,464^{* *}$ & $-4,932 * *$ \\
\hline$D^{8: 32} \cdot R_{N F}$ & $-0,549$ & $-0,510$ & $-0,435$ \\
\hline$r_{t-1}$ & $-0,091 * * *$ & $-0,091 * * *$ & $-0,091 * * *$ \\
\hline$r_{t-2}$ & 0,000 & 0,000 & 0,000 \\
\hline \multicolumn{4}{|l|}{ Variance equation } \\
\hline cons & $0,436 * * *$ & $0,437 * * *$ & $0,436 * * *$ \\
\hline$\varepsilon_{t-1}^{2}$ & $0,146 * *$ & $0,143 * *$ & $0,146 * *$ \\
\hline$\varepsilon_{t-2}^{2}$ & $0,059 * * *$ & $0,059 * * *$ & $0,058 * * *$ \\
\hline$\varepsilon_{t-3}^{2}$ & $0,035 * * *$ & $0,035 * * *$ & $0,035 * * *$ \\
\hline $\mathrm{LL}$ & $-7974,53$ & $-7982,74$ & $-7974,38$ \\
\hline $\mathrm{BIC}$ & 2,2419 & 2,2441 & 2,2431 \\
\hline LR-Test against model (A) & $92,32 * * *$ & $75,90^{* * *}$ & $92,62^{* * *}$ \\
\hline LR-Test against model (C) & & & $25,82 * * *$ \\
\hline LR-Test against model (F) & $68,02 * * *$ & $51,59 * * *$ & $68,31^{* * *}$ \\
\hline LR-Test against model (G) & $16,92 * * *$ & 0,50 & $17,21^{* * *}$ \\
\hline LR-Test against model (H) & $13,98 * * *$ & & $14,27 * * *$ \\
\hline LR-Test against model (I) & & $38,51 * * *$ & $55,22 * * *$ \\
\hline LR-Test against model $(\mathrm{J})$ & & & 0,29 \\
\hline LR-Test against model (K) & & & $16,71^{* * *}$ \\
\hline
\end{tabular}


TABLE 2 (continued)

QML estimation of $\mathrm{AR}(2)-\mathrm{ARCH}(3)$ models for two-min log returns during the intraday interval 8:22-9:52 a.m. EST on employment announcement days for which no other macroeconomic report is released at the same time. The sample period is Jan. 1991 - Dec. 2005, resulting in 7245 observations (i.e. 161 days with no overlapping announcements $\times 452$-min intervals).

The estimated model for log returns $r_{t}$ is given by $r_{t}=c+\sum_{j=1}^{2} \phi_{j} r_{t-j}+x_{t}^{\prime} \beta+\varepsilon_{t}$, where $\varepsilon_{t} \sim N\left(0, h_{t}\right)$, $t$ indexes the first interval after the announcement, 8:30-8:32 a.m., $x_{t}$ denotes a vector of explanatory variables and $\beta$ is the corresponding coefficient vector. $h_{t}$ is given by $h_{t}=\omega+\sum_{j=1}^{3} \psi_{j} \varepsilon_{t-j}^{2}+s_{t}$, where $s_{t}=\delta^{s} \cdot \bar{t}+\sum_{j=1}^{5}\left(\delta_{c, j}^{s} \cos (j \cdot \bar{t} \cdot 2 \pi)+\delta_{s, j}^{s} \sin (j \cdot \bar{t} \cdot 2 \pi)\right)$ denotes the seasonality function based on the parameters $\delta^{s}, \delta_{c, j}^{s}, \delta_{s, j}^{s}$ and a normalized time trend $\bar{t} \in[0,1]$ given by the elapsed time (in minutes) in the interval 8:22 to 9:52 a.m. divided by 90 . The estimated seasonality parameters are omitted in the table.

The regressors $x_{t}$ are the surprise in U.S. nonfarm payrolls, $S_{N F}$, and in unemployment rates, $S_{U N}$, as well as revisions of nonfarm payrolls $R_{N F}$ interacted with time dummies indicating the intervals 8:28-8:30 a.m. $\left(D^{8: 28}\right), 8: 30-8: 32$ a.m. $\left(D^{8: 30}\right)$ and 8:32-8:34 a.m. $\left(D^{8: 32}\right)$. Surprises are computed based on U.S. employment report figures released by the BLS and consensus forecasts provided by Informa Global Markets (IGM), formerly MMS. The variables $S_{N F}$ are included as polynomials up to order 3 and interact with dummy variables $D^{\pi \text { high }}\left(D^{\pi \text { low }}\right)$ which takes on the value 1 if estimated price-response coefficient $\hat{\pi}_{m}$ at month $m$ is higher (lower) than its sample median, and 0 otherwise. $\hat{\pi}_{m}$ is given by $\hat{\pi}_{m}=\hat{\rho}_{A, m} /\left(\hat{\rho}_{F, m}+\hat{\rho}_{A, m}\right)$, where $\hat{\rho}_{A, m}=1 / \hat{g}_{m+1 \mid m}, \hat{g}_{m+1 \mid m}$ is the one-step-ahead prediction of the conditional variance of (percentage) revision of the nonfarm payroll figure in month $m, \dot{R}_{N F, m}$, computed based on rolling sample ARMA-GARCH models for the time series of historical revisions, and $\hat{\rho}_{F, m}=1 / \hat{s}_{F, m}^{2}$ with $\hat{s}_{F, m}$ denoting the cross-sectional standard deviation of IGM forecasts for the employment release for a particular month $m$.

The table reports the log likelihood (LL), the Bayes information criterion (BIC) and $\chi^{2}$ statistics of LR tests on the inequality of individual parameters. Statistical inference is based on QML standard errors (Bollerslev and Wooldridge 1992). ${ }^{* * *},{ }^{* *}$, and ${ }^{*}$ indicates significance at the $1 \%, 5 \%$, and $10 \%$ level, respectively. Except for the LR tests, the level of significance is based on two-sided tests. 


\section{References}

Abarbanell, Jeffrey S., William N. Lanen, and Robert E. Verrecchia. 1995. "Analysts' Forecasts as Proxies for Investor Beliefs in Empirical Research." Journal of Accounting and Economics, 20 (1): 31-60.

Almeida, Alvara, Charles A. E. Goodhart, and Richard Payne. 1998. "The Effects of Macroeconomic News on High Frequency Exchange Rate Behavior." Journal of Financial and Quantitative Analysis, 33 (3): 383-408.

Andersen, Torben G., and Tim Bollerslev. 1998. "Deutsche Mark-Dollar Volatility: Intraday Activity Patterns, Macroeconomic Announcements and Longer Run Dependencies." Journal of Finance, 53 (1): 219-265.

Andersen, Torben G., Tim Bollerslev, Francis X. Diebold, and Clara Vega. 2003. "Micro Effects of Macro Announcements: Real-Time Price Discovery in Foreign Exchange." American Economic Review, 93 (1): 38-62.

Balduzzi, Pierluigi, Edwin J. Elton, and T. Clifton Green. 2001. "Economic News and Bond Prices: Evidence from the U.S. Treasury Market." Journal of Financial and Quantitative Analysis, 36 (4): 523-543.

Becker, Kent G., Joseph E. Finnerty, and Kenneth J. Kopecky. 1996. "Macroeconomic News and the Efficiency of International Bond Futures Markets." Journal of Futures Markets, 16 (2): 131-145.

Bollerslev, Tim, and Jeffrey Wooldridge. 1992. "Quasi-Maximum Likelihood Estimation and Inference in Dynamic Models with Time Varying Covariances." Econometric Reviews, 11 (2): 143-172.

Boyd, John H., Jian Hu, and Ravi Jagannathan. 2005. "The Stock Market's Reaction to Unemployment News: Why Bad News is Usually Good for Stocks." Journal of Finance, 60 (2): 649-673. 
Conrad, Jennifer, Bradford Cornell, and Wayne R. Landsman. 2002. "When Is Bad News Really Bad News?" Journal of Finance, 57 (6): 2507-2533.

Feltham, Gerald A., and Jinhan Pae. 2000. "Analysis of the Impact of Accounting Accruals on Earnings Uncertainty and Response Coefficients." Journal of Accounting, Auditing and Finance, 15 (3): 199-220.

Fleming, Michael J., and Eli M. Remolona. 1999. "Price Formation and Liquidity in the U.S. Treasury Market: The Response to Public Information." Journal of Finance, 54 (5): 1901-1915.

. 1999. "The Term Structure of Announcement Effects." Federal Reserve Bank of New York, Staff Reports 67.

. 1999. "What Moves Bond Prices." Journal of Portfolio Management, 25 (4): $28-38$.

Hardouvelis, Gikas A. 1988. "Economic News, Exchange Rates, and Interest Rates." Journal of International Money and Finance, 7 (1): 23-35.

Hautsch, Nikolaus, and Dieter Hess. 2002. "The Processing of Non-anticipated Information in Financial Markets: Analyzing the Impact of Surprises in the Employment Report." European Finance Review, 6 (2): 133-161.

. 2007. "Bayesian Learning in Financial Markets: Testing for the Relevance of Information Precision in Price Discovery." Journal of Financial and Quantitative Analysis, 42 (1): 189-208.

Hess, Dieter. 2004. "Determinants of the Relative Price Impact of Unanticipated Information in U.S. Macroeconomic Releases." Journal of Futures Markets, 24 (7): 609-630.

Mattsson, Lars-Göran, Mark Voorneveld, and Jörgen W. Weibull. 2007. "Better may be Worse: Some Monotonicity Results and Paradoxes in Discrete Choice." Theory and Decision, 63 (2): 121-151. 
Milgrom, Paul 1981. "Good News and Bad News: Representation Theorems and Applications." Bell Journal of Economics, 23: 380-391.

Mohammed, Suleiman R., and Pradeep K. Yadav. 2002. "Quality of Information and Volatility Around Earnings Announcements," Technical Report, University of Strathclyde.

Ottaviani, Marco, and Peter Norman Sorensen. 2006. "The Strategy of Professional Forecasting." Journal of Financial Economics, 81 (2): 441-466.

Pilotte, Eugene, and Timothy Manuel. 1996. "The Market's Response to Recurring Events: The Case of Stock Splits." Journal of Financial Economics, 41 (1): $111-127$.

Richardson, Scott A., Richard G. Sloan, Mark T. Soliman, and A. Irem Tuna. 2005. "Accrual Reliability, Earnings Persistence and Stock Prices." Journal of Accounting and Economics, 39 (3): 437-485.

Sloan, Richard G. 1996. "Do Stock Prices Fully Reflect Information in Accruals and Cash Flows About Future Earnings." Accounting Review, 71 (3): 289-315.

Subramanyam, K. R. 1996. "Uncertain Precision and Price Reactions to Information." Accounting Review, 71 (2): 207-220. 


\section{CFS Working Paper Series:}

\begin{tabular}{|c|c|c|}
\hline No. & Author(s) & Title \\
\hline $2008 / 27$ & $\begin{array}{l}\text { Francis X. Diebold } \\
\text { Canlin Li } \\
\text { Vivian Z. Yue }\end{array}$ & $\begin{array}{l}\text { Global Yield Curve Dynamics and Interactions: A } \\
\text { Dynamic Nelson-Siegel Approach }\end{array}$ \\
\hline $2008 / 26$ & $\begin{array}{l}\text { Francis X. Diebold } \\
\text { Kamil Yilmaz }\end{array}$ & $\begin{array}{l}\text { Measuring Financial Asset Return and Volatilty } \\
\text { Spillovers, with Application to Global Equity } \\
\text { Markets }\end{array}$ \\
\hline $2008 / 25$ & $\begin{array}{l}\text { Günter W. Beck } \\
\text { Volker Wieland }\end{array}$ & $\begin{array}{l}\text { Central Bank Misperceptions and the Role of } \\
\text { Money in Interest Rate Rules }\end{array}$ \\
\hline $2008 / 24$ & $\begin{array}{l}\text { Christos Koulovatianos } \\
\text { Carsten Schröder } \\
\text { Ulrich Schmidt }\end{array}$ & $\begin{array}{l}\text { Confronting the Robinson Crusoe paradigm with } \\
\text { household-size heterogeneity }\end{array}$ \\
\hline $2008 / 23$ & $\begin{array}{l}\text { Jin Cao } \\
\text { Gerhard Illing }\end{array}$ & Endogenous Systemic Liquidity Risk \\
\hline $2008 / 22$ & $\begin{array}{l}\text { Zeno Enders } \\
\text { Gernot J. Müller } \\
\text { Almut Scholl }\end{array}$ & $\begin{array}{l}\text { How do Fiscal and Technology Shocks affect Real } \\
\text { Exchange Rates? New Evidence for the United } \\
\text { States }\end{array}$ \\
\hline $2008 / 21$ & Miriam Sperl & $\begin{array}{l}\text { Quantifying the Efficiency of the Xetra LOB } \\
\text { Market. A Detailed Recipe }\end{array}$ \\
\hline $2008 / 20$ & $\begin{array}{l}\text { Maria Concetta Chiuri } \\
\text { Tullio Jappelli }\end{array}$ & $\begin{array}{l}\text { Do the elderly reduce housing equity? An } \\
\text { international comparison }\end{array}$ \\
\hline 2008/19 & Annamaria Lusardi & $\begin{array}{l}\text { Financial Literacy: An Essential Tool for Informed } \\
\text { Consumer Choice? }\end{array}$ \\
\hline 2008/18 & Annamaria Lusardi & $\begin{array}{l}\text { Increasing the Effectiveness of Financial Education } \\
\text { in the Workplace }\end{array}$ \\
\hline
\end{tabular}

Copies of working papers can be downloaded at http://www.ifk-cfs.de 